\title{
La sculpture de l'Antiquité tardive et du haut Moyen Âge en péninsule Ibérique, une révision nécessaire
}

Late Antique and Early Medieval Sculpture in the Iberian Peninsula, a necessary revision

\section{Gisela Ripoll}

\section{OpenEdition}

\section{Journals}

Édition électronique

URL : https://journals.openedition.org/cel/19054

DOI : 10.4000/cel.19054

ISSN : 2262-208X

\section{Éditeur}

École du Louvre

\section{Référence électronique}

Gisela Ripoll, «La sculpture de l'Antiquité tardive et du haut Moyen Âge en péninsule Ibérique, une révision nécessaire ", Les Cahiers de l'École du Louvre [En ligne], 17 | 2021, mis en ligne le 18 novembre 2021, consulté le 13 décembre 2021. URL : http://journals.openedition.org/cel/19054 ; DOI : https:// doi.org/10.4000/cel.19054

Ce document a été généré automatiquement le 13 décembre 2021.

\section{c)}

Les Cahiers de l'École du Louvre sont mis à disposition selon les termes de la licence Creative Commons Attribution - Pas d'Utilisation Commerciale - Pas de Modification 4.0 International. 


\section{La sculpture de l'Antiquité tardive et du haut Moyen Âge en péninsule Ibérique, une révision nécessaire}

Late Antique and Early Medieval Sculpture in the Iberian Peninsula, a necessary revision

\section{Gisela Ripoll}

\section{In memoriam}

Jordi Casanovas (1953-2020)

Manuel Sotomayor (1922-2020)

Juan A. Souto (1959-2011)

1 Le 23 juillet 2020, tard dans la nuit, j'ai appris avec une profonde émotion que la vie du Père Manuel Sotomayor s'était éteinte la nuit précédente. Il a vécu presque un siècle. Il avait 97 ans. Au même moment, je travaillais sur ce texte à propos de la sculpture ${ }^{1}$, sujet qu'il connaissait bien, puisqu'il en était l'un des pionniers.

2 Les contributions du Père Sotomayor à l'histoire et à l'archéologie du christianisme et de l'Antiquité tardive en péninsule Ibérique sont majeures dans tous les domaines histoire de l'Église, histoire de Grenade, territoire et paroisses, iconographie chrétienne, archéologie des villae, céramique sigillée, et tant d'autres choses - mais il faut citer ici les "fers de lance " les plus importants pour la sculpture funéraire hispanique, les deux volumes sur les sarcophages paléochrétiens publiés dans les années $1970^{2}$.

Ces deux livres forment un point de départ fondamental, après la publication initiale de Giuseppe Bovini, Sarcofagi paleocristiani della Spagna de 1954. Sotomayor a donné les clés pour la typologie, la classification, la chronologie et l'iconographie des sarcophages chrétiens hispaniques. 
C'est donc avec un profond respect envers le Père Sotomayor et nos prédécesseurs que je me permets de partager avec vous quelques questions qui montrent quels sont, de mon point de vue, les «pourquoi » et quelques-uns des " grands problèmes » de la sculpture hispanique.

\section{Pionniers et précurseurs}

5 La découverte de Segobriga (Cabeza de Griego, Saelices, Cuenca) a marqué un tournant dans l'historiographie hispanique. À Segobriga, une église funéraire a été mise au jour avec toute une série d'inscriptions épiscopales, dont certaines versifiées, des sculptures décoratives et du mobilier liturgique du VII ${ }^{e}$ siècle. Le site, connu depuis le XVI ${ }^{e}$ siècle, a été l'objet de multiples recherches à partir du milieu du XVIII ${ }^{e}$ siècle, avec José Altisent. Pratiquement tous les savants se sont intéressés à l'identification de Segobriga et de son siège épiscopal, depuis Enrique Flórez à Fidel Fita, Tomás de Torres y Moya, Gregorio Mayans y Siscar, ce qui a conduit la Real Academia de la Historia, en 1793, à envoyer José Cornide comme commissaire pour identifier les éléments découverts ${ }^{3}$. La bibliographie sur Segobriga est très vaste ${ }^{4}$, mais la sculpture n'avait pas fait l'objet d'une étude détaillée - bien que ce fût absolument nécessaire - jusqu'à très récemment, lorsque cela a été réalisé par Jenny Abura ${ }^{5}$.

6 La recherche faite dans la deuxième moitié du $\mathrm{XX}^{\mathrm{e}}$ siècle s'appuie sur le travail réalisé par les archéologues, mais aussi par les historiens de l'art et architectes. De notables découvertes et contributions jalonnent la fin $\mathrm{du}_{\mathrm{XIX}} \mathrm{X}^{\mathrm{e}}$ et la première moitié $\mathrm{du} \mathrm{XX}^{\mathrm{e}}$ siècle, parmi lesquelles je signale seulement celles qui, à mon sens, sont des « faits marquants ${ }^{6} »$.

7 Les Monumentos Arquitectónicos de España (1859-1881), sous l'égide de l'Académie royale des Beaux-Arts de San Fernando, avait pour objectif de faire connaître les principaux monuments architecturaux espagnols ainsi que certains objets d'art ou éléments décoratifs ${ }^{7}$. La sélection des édifices est arbitraire, de même que leur place dans les différents volumes. À cette œuvre magistrale ont participé, entre autres, José Amador de los Ríos, Rodrigo Amador de los Ríos, Manuel de Assas, Pedro de Madrazo et Aureliano Fernández Guerra, accompagnés de dessinateurs hors pair. On trouve chez Aureliano Fernández Guerra un intérêt manifeste pour l'archéologie chrétienne, l'iconographie funéraire des sarcophages et l'épigraphie. Plus généralement, l'archéologie chrétienne et la sculpture tardive ont bien trouvé leur place dans ces volumes. Bien qu'il s'insère dans une étude des "monuments", le décor architectonique devient une œuvre d'art pratiquement isolée de son contexte d'origine, malgré cette attention initiale portée à l'archéologie qui, elle, permet effectivement d'établir des contextes. L'entreprise des Monumentos Arquitectónicos de España a conduit à la création d'une nouvelle initiative, peut-être moins ambitieuse en termes de contenu détaillé, mais également spectaculaire et attrayante pour les lecteurs cultivés de l'époque. Il s'agit du Museo Español de Antigüedades, une publication périodique de grand format éditée en 11 volumes entre 1872 et 1880 et dirigée par Juan de Dios de la Rada y Delgado. L'entreprise avait un caractère de vulgarisation pour le grand public, mais en raison des insignes collaborateurs, issus de l'Académie royale et du Musée Archéologique National, plusieurs de ces articles sont devenus d'importantes contributions à la recherche et ses illustrations sont d'une grande valeur (fig. 1). 
Fig. 1

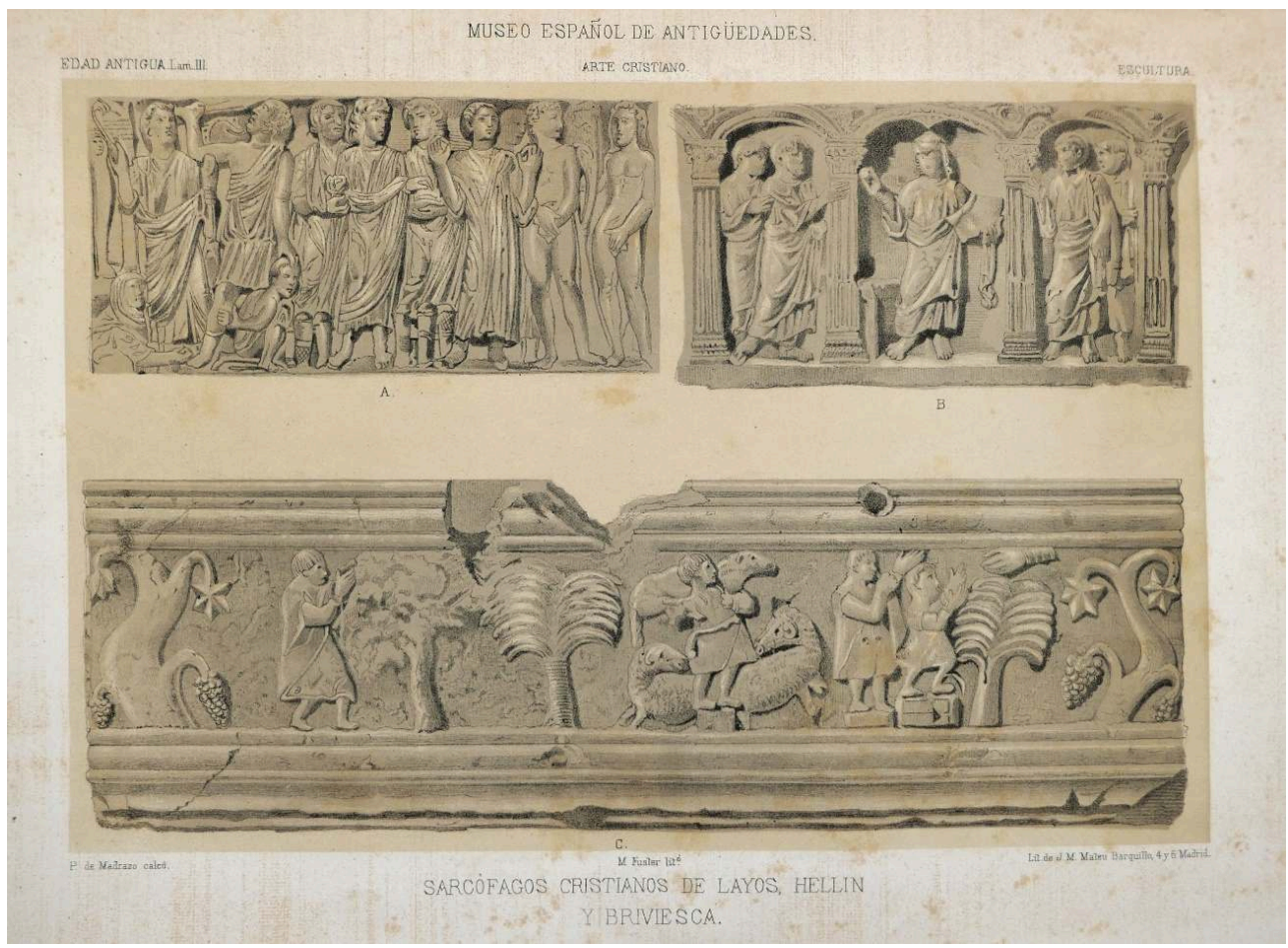

Planche gravée du Museo Español de Antigüedades (Edad Antigua, lám. III, Arte Cristiano, Escultura), Sarcophages chrétiens de Layos (Tolède), Hellín (Albacete) et Briviesca (La Bureba, Burgos). Chalcographie P. de Madrazo, lithographie M. Fuster, impression lithographique M. Mateu Barquillo (Collection personnelle de J. Casanovas).

(C) G. Ripoll

8 La découverte du Trésor de Guarrazar (Huerta de Guarrazar, Tolède), révélé en 1858, est un des grands moments historiographiques. L'Académie envoya José Amador de los Ríos faire des fouilles supplémentaires après la découverte du trésor. Le fruit de ce travail est son superbe et encore indispensable ouvrage publié en $1861:$ El arte latinobizantino en España y las coronas visigodas de Guarrazar ${ }^{9}$. On disposait pour la première fois de toute une série de matériels distincts, dans un contexte du VII ${ }^{\mathrm{e}}$ siècle. D'un côté l'édifice ecclésiastique, oratoire ou mausolée, qui est aujourd'hui interprété comme une église à plan basilical et à trois nefs ${ }^{10}$. De l'autre, le décor sculpté (fig. 2), les ornamenta et instrumenta à caractère votif, très probablement cachés face à la menace de la prise de Tolède par les Arabes en 712, et l'épigraphie, sur des objets votifs mais aussi avec l'inscription funéraire de Crispinus datée de $693^{11}$. 
Fig. 2

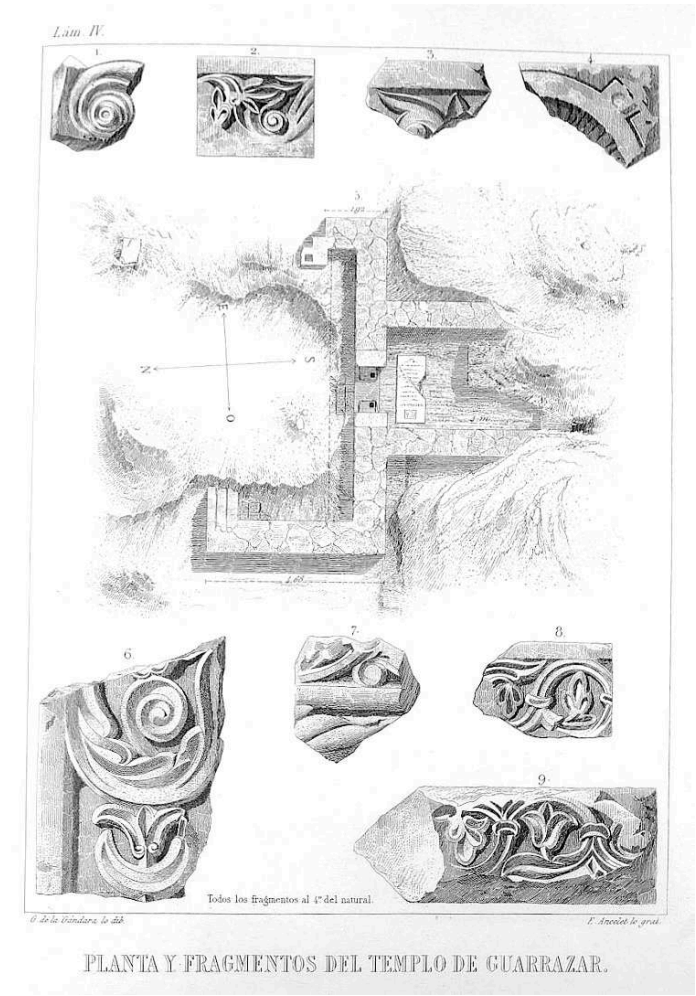

Guarrazar (Tolède), planimétrie et fragments sculptés. Planche IV de José Amador de los Ríos, El arte latino-bizantino en España y las coronas visigodas de Guarrazar, 1861 (op. cit. note 9).

(c) G. Ripoll

Le changement de siècle comporta la création d'un ambitieux projet, le catálogo Monumental de España (1900-1961), auquel presque tous les historiens de l'art et architectes ont participé. Il s'agit d'un catalogue/inventaire d'architecture, et donc de l'ornementation architectonique sculptée, ainsi que des objets. Un travail très méthodique et bien organisé permet aujourd'hui de consulter le catalogue en ligne ${ }^{12}$. Le Projet SATHMA, centré sur les fonds conservés du Recueil général des monuments sculptés en France pendant le haut Moyen Âge (IV ${ }^{e} X^{e}$ siècles) au Musée du Louvre, qui est présenté dans ce même volume par Arnaud Millereux et Vincent Rousseau, montre beaucoup de ressemblances avec le Catálogo Monumental. Aussi bien dans l'un que dans l'autre existent de nombreuses données primaires, telles que photographies, carnets, notes, dessins, croquis et clichés, qui obligent à la numérisation pour constituer un dépôt consultable en ligne destiné aux chercheurs mais aussi à tout le public cultivé.

Deux publications ont été fondamentales pour la période qui nous intéresse. D’abord celle de Josep Puig i Cadafalch à propos de l'architecture romane de la Catalogne, l'un de ces "mythes " de l'historiographie ${ }^{13}$. Il s'agit de trois volumes, dont les deux premiers intéressent directement l'objet de cette présentation. Le volume I porte sur l'architecture romaine et chrétienne préromane et le II traite l'architecture romane jusqu'à la fin du XI $\mathrm{XI}^{\mathrm{e}}$ siècle $^{14}$. À la même période remonte le livre de Manuel Gómez Moreno sur les églises mozarabes, résultat culminant de toutes ses recherches et de la découverte des églises attribuées à l'époque wisigothique, son Iglesias Mozárabes de 1919. 
11 La connaissance de toute cette architecture, avec des attributions chronologiques très claires pour les auteurs cités, a permis de travailler sur le décor sculpté ${ }^{15}$. Il faut rappeler enfin l'ouvrage posthume de Puig i Cadafalch, L'Art wisigothique et ses survivances ${ }^{16}$, construit, surtout, à partir des cours qu'il avait prononcés à la Sorbonne pendant son exil et grâce à la Fondation Cambó, dont la rédaction date des années 1937-1940. Il est considéré par maints chercheurs comme un livre émouvant ${ }^{17}$.

\section{La construction de la discipline}

Une fois les bases établies, une nouvelle génération de chercheurs est venue défendre l'Antiquité tardive comme une période importante en elle-même, ainsi que la présence des Wisigoths qui a indubitablement marqué la réalité matérielle, tout cela sans oublier le fort impact que pouvaient avoir les Byzantins dans le sud-est de la péninsule.

Parmi les premiers travaux, figure celui d'Emilio Camps Cazorla (1903-1952), publié dans le cadre de l'éminente Historia de España dirigée par Ramón Menéndez Pidal, issu de la formation que lui a offert Gómez Moreno dans cette discipline ${ }^{18}$.

Au même moment, les recherches de Helmut Schlunk (1906-1982) commencent à voir le jour, à mi-chemin entre les traditions historiographiques émergentes du début du siècle et la mise en place des grands problèmes de l'architecture, de la sculpture et de la liturgie posés par la recherche au milieu du siècle ${ }^{19}$.

16 Très tôt, Schlunk a attiré l'attention sur les particularités de la sculpture hispanique des $\mathrm{VI}^{\mathrm{e}}$ et $\mathrm{VII}^{\mathrm{e}}$ siècles et sur ses étroites relations avec le monde méditerranéen et byzantin ${ }^{20}$. Ses contributions sur l'art wisigothique et l'art asturien dans les volumes d'Ars Hispaniae, qui ont connu une large diffusion scientifique, mais aussi auprès du grand public, peuvent être considérées comme des jalons historiographiques de la discipline ${ }^{21}$. Ce travail a culminé dans le monumental Die Denkmäler der frühchristlichen und westgotischen Zeit de $1978^{22}$, de même que dans ses travaux sur Tolède et les Asturies $^{23}$, d'une pleine maturité, pour ne citer que quelques exemples de sa grande production bibliographique.

Les années centrales du $\mathrm{XX}^{\mathrm{e}}$ siècle sont marquées par la figure de Pere de Palol (1923-2005), qui s'insère pleinement dans la discipline archéologique de la période, utilisant la fouille et la stratigraphie pour élucider les problèmes posés par l'histoire de l'art, comme dans les fouilles de l'église de San Juan de Baños à Palencia (fig. 3). Ses travaux archéologiques étaient très diversifiés, allant de l'archéologie funéraire à l'architecture, du monde urbain au monde rural, de l'architecture civile publique à l'architecture ecclésiastique, en passant par les sarcophages, le mobilier liturgique, les analyses de la production de métaux, les typologies d'objets, etc. Palol avait besoin d'aborder tous les sujets afin d'objectiver sa caractérisation de la période et de contribuer avec ses résultats à une plus grande connaissance. Mise en perspective, la contribution de Palol est significative en ce qu'elle ouvre différentes voies de recherche et pose ainsi toute une série de problèmes qui se sont progressivement précisés, ou ont été écartés. 
Fig. 3

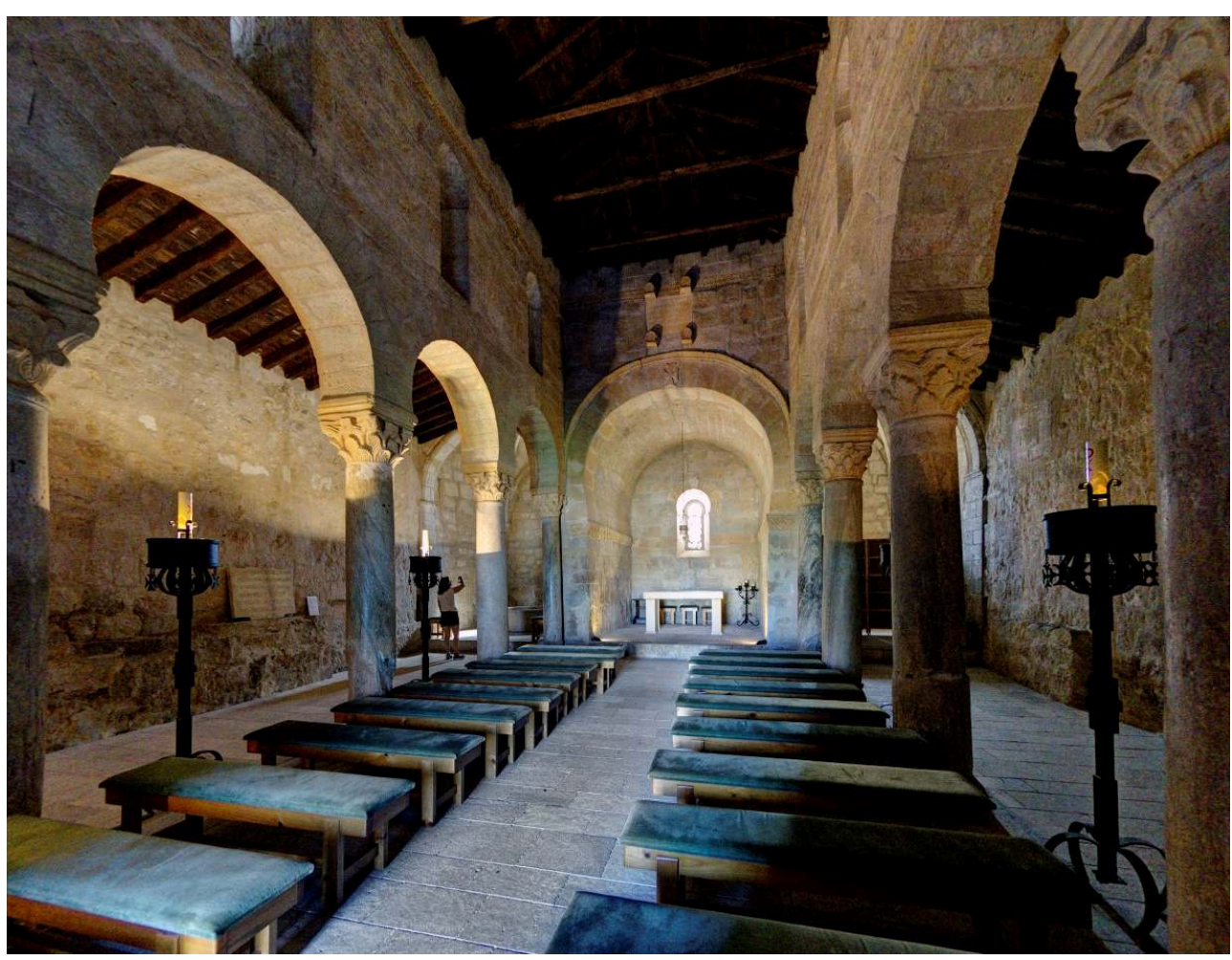

San Juan de Baños (Palencia).

(C) Á.M. Felicísimo

Il est impossible d'évoquer ici toutes ses contributions importantes, mais il convient peut-être de mentionner celle présentée au Centre de Spolète en $1956^{24}$, où il a exposé ce qu'il considérait comme les productions de la période wisigothique, en mettant l'accent sur les influences germaniques, réservées aux objets de parure personnelle découverts dans les nécropoles du plateau castillan du $\mathrm{VI}^{\mathrm{e}}$ siècle, et les influences du monde romain, comme substrat commun à toute la Méditerranée; et ce par opposition au byzantinisme défendu par Helmut Schlunk, comme cela a déjà été dit. Et je ne peux pas ne pas mentionner ce qui a été, et est toujours, le livre de chevet de nombreux chercheurs, son Arqueología cristiana de la España romana de $1967^{25}$, qui a rassemblé pour la première fois toutes les preuves matérielles du début du christianisme hispanique et qui était fondé sur des études détaillées publiées par lui-même ${ }^{26}$. Il en est ressorti deux ouvrages destinés au grand public, sur l'art paléochrétien et l'art wisigothique ${ }^{27}$. Et, je crois que deux publications sont fondamentales pour connaitre la contribution de Palol à l'Hispanie de l'Antiquité tardive. L'une, de synthèse, sur l'art et l'archéologie, au sein de la réédition de l'Histoire de l'Espagne de Menéndez Pidal ${ }^{28}$. Et la grande entreprise qu'il a réalisée avec Antoni Pladevall, considérée comme le numéro zéro de la grande encyclopédie Catalunya Romànica ${ }^{29}$.

19 Il est important de rappeler qu'à la fin des années 1960, José Vives fournit des instruments de travail essentiels pour la connaissance de l'histoire hispanique du IV au VIII siècle, extrêmement utiles pour les archéologues et les historiens de l'art. Il publie le recueil des inscriptions et le corpus conciliaire ${ }^{30}$. L'une des toutes premières contributions de Thilo Ulbert coïncide avec ces dates mais est passée pratiquement inaperçue des chercheurs ${ }^{31}$. 

Duval (1929-2018), qui a établi des liens très étroits avec les chercheurs catalans en participant à de nombreuses initiatives et en proposant des lectures et solutions toujours éclairantes aux problèmes posés par l'architecture, la sculpture et la liturgie hispaniques ${ }^{32}$. Et tant d'autres chercheurs, aussi bien avec des contributions majeures que mineures, qu'hélas, je ne peux toutes citer. Ils étaient tous immergés dans l'univers de l'archéologie paléochrétienne et de l'Antiquité tardive, avec la ferme conviction que l'archéologie hispanique devait encore bâtir ses inventaires et catalogues.

C'est justement au moment où l'on commence à construire les instruments de travail essentiels pour la caractérisation de la période qu'il faut rappeler les deux volumes de Manuel Sotomayor à propos des sarcophages, cités tout à fait au début de ce texte ${ }^{33}$.

Les corpora hispaniques commencent à émerger, plaçant presque toujours l'architecture devant la sculpture, mais sans oublier cette dernière. L'un de ces projets de publication les plus titanesques est probablement celui de la Catalunya Romànica ${ }^{34}, 27$ volumes de grand format, précédés par un numéro zéro, concernant l'Antiquité tardive et le haut Moyen Âge, déjà cité ${ }^{35}$. Il faut enfin signaler que Xavier Barral avait déjà publié un corpus sur l'art préroman catalan ${ }^{36}$, qui facilite, d'un certain point de vue, la mise en place du sujet et dont la consultation est encore nécessaire aujourd'hui. Des entreprises similaires, mais moins ambitieuses ont été mises en place dans d'autres régions, comme les Asturies ${ }^{37}$.

\section{De la fin du $X X^{\mathrm{e}}$ siècle au début du $X X I^{\mathrm{e}}$ siècle}

L'ensemble de ces recherches ouvre la voie aux nouvelles générations qui intègrent dans leur travail la nécessité de construire des références fermes, des catalogues, des inventaires, avec des points de repères chronologiques fondés sur l'architecture et l'archéologie.

24 Je voudrais citer quelques auteurs et leurs publications, puisqu'ils marquent un point d'inflexion dans l'étude de la sculpture de l'Antiquité tardive et du haut Moyen Âge en péninsule Ibérique, tout en signalant que nous ne disposons pas de corpora de sculpture comme il en existe pour d'autre pays, tels le corpus italien publiée par Spolète, le Recueil de la Gaule, le corpus de l'Angleterre, la Suisse, etc., qui sont la base pour la reprise numérique, comme on l'a vu dans les journées d'étude de septembre 2020 et comme on le constate dans la présente publication.

Le corpus de Mérida de Cruz Villalón publié en $1985^{38}$ marque un avant et un après dans la connaissance du matériel sculpté et on peut le considérer comme le premier corpus d'une des collections les plus importantes de péninsule Ibérique : celle d'Emerita Augusta, capitale de la diocesis Hispaniarum. Il a été précédé d'une étude qui n'est pas passée inaperçue mais qui n'a pas été suffisamment mise en valeur, celle d'Isabel Zamorano, qui rassemble tout le matériel de Tolède ${ }^{39}$.

26 En parallèle des travaux sur Mérida, Xavier Barral i Altet a souligné toute une série de besoins, dans une analyse qui est encore totalement valable aujourd'hui, même s'il semble qu'elle soit passée inaperçue : un premier article publié dans les Corsi di Ravenna de $1987^{40}$ et sa réflexion élaborée dans le cadre des "Réunions d'archéologie paléochrétienne ", en l'occurrence celle de Mahon de 1988, organisée par P. de Palol et J. M. Gurt ${ }^{41}$. Xavier Barral y a revendiqué la nécessité des points suivants ${ }^{42}$ : 
l'établissement de corpora par régions, la publication des contextes archéologiques des sculptures trouvées, des critères chronologiques des ateliers et des productions au-delà $\mathrm{du}$ style, un usage correct du vocabulaire et donc une meilleure spécification des différentes fonctions du matériel sculpté, l'inclusion de pièces en céramique et en stuc, en plus de la pierre et du marbre, et un meilleur rapport de la décoration figurative, mais également géométrique et végétale, et des productions de mosaïques, avec les sculptures, tout cela dans le but de répondre à la connaissance des circuits de production et de commercialisation en fonction d'une chronologie plus précise.

Jean-Marie Hoppe, parallèlement aux études citées, s'est plongé dans l'analyse des productions sculptées de la péninsule aux $\mathrm{VI}^{\mathrm{e}}$ et $\mathrm{VII}^{\mathrm{e}}$ siècles, à partir de l'histoire de l'art, et surtout de l'esthétique et l'iconographie, afin de décortiquer les problèmes du " wisigothisme » local et des influences byzantines ${ }^{43}$. Ses premiers travaux ont été suivis peu de temps après par une nouvelle et importante réflexion publiée dans Visigodos $y$ omeyas en $2000^{44}$.

Presque dix ans après la parution du volume consacré à Mérida par Cruz Villalón, Noël Duval a dirigé une thèse de doctorat, celle de Rafaële Mourgues ${ }^{45}$, que j'ai suivie de très près à Madrid et à Barcelone, portant sur l'ensemble de la sculpture wisigothique hispanique, thèse restée inédite, mais qui a donné lieu à un article, à mon sens de grande importance à ce moment-là, car il posait les problèmes de cette sculpture et proposait des méthodologies, des analyses ainsi que des résultats, novateurs ${ }^{46}$.

C'est dans les années 1990 que Luis Caballero, l'un des principaux chercheurs dans ce domaine de l'architecture, du CSIC, le " CNRS espagnol ", a établi un lien entre les caractéristiques formelles de la sculpture wisigothique et celles des sculptures réalisées sous les Omeyyades en Syrie et qu'il a ramené la chronologie de l'architecture, de la sculpture et de l'épigraphie aux VIII ${ }^{e}$ et IX ${ }^{e}$ siècles, voire au $\mathrm{X}^{\mathrm{e}}$ siècle ${ }^{47}$. Ce n'est pas ici le lieu pour discuter l'ensemble du problème, mais je veux donner quelques éléments de la discussion car ils touchent directement la sculpture architectonique et décorative, notamment en ce qui concerne l'architecture ecclésiastique. Caballero, en suivant l'hypothèse de Sally Garen ${ }^{48}$, a proposé de voir des correspondances entre les restes fragmentaires de la sculpture en stuc à décor de rinceau stylisé orné de fleurons à huit pétales de Santa María de Melque (Tolède) (fig. 4) et ceux du palais omeyyade de Khirbat al-Mafjar (au nord de Jéricho, en Palestine), pour lesquels le califat omeyyade de Cordoue aurait servi de voie de transmission. Le problème majeur s'est posé en passant de cet exemple à l'étude de l'ensemble de l'architecture dite wisigothique, essentiellement les édifices qui constituent la " pointe de l'iceberg »: Santa María de Melque (Tolède), San Juan de Baños (Palencia), Quintanilla de las Viñas (Burgos) (fig. 5) et Santa Comba de Bande (Orense). Devant l'absence de structures voûtées en pierre des édifices hispano-musulmans et alors qu'une tradition de voûtes est visible dans les églises d'époque wisigothique, la datation fut donc retardée afin que l'ensemble du puzzle retrouve une cohérence ${ }^{49}$. 
Fig. 4

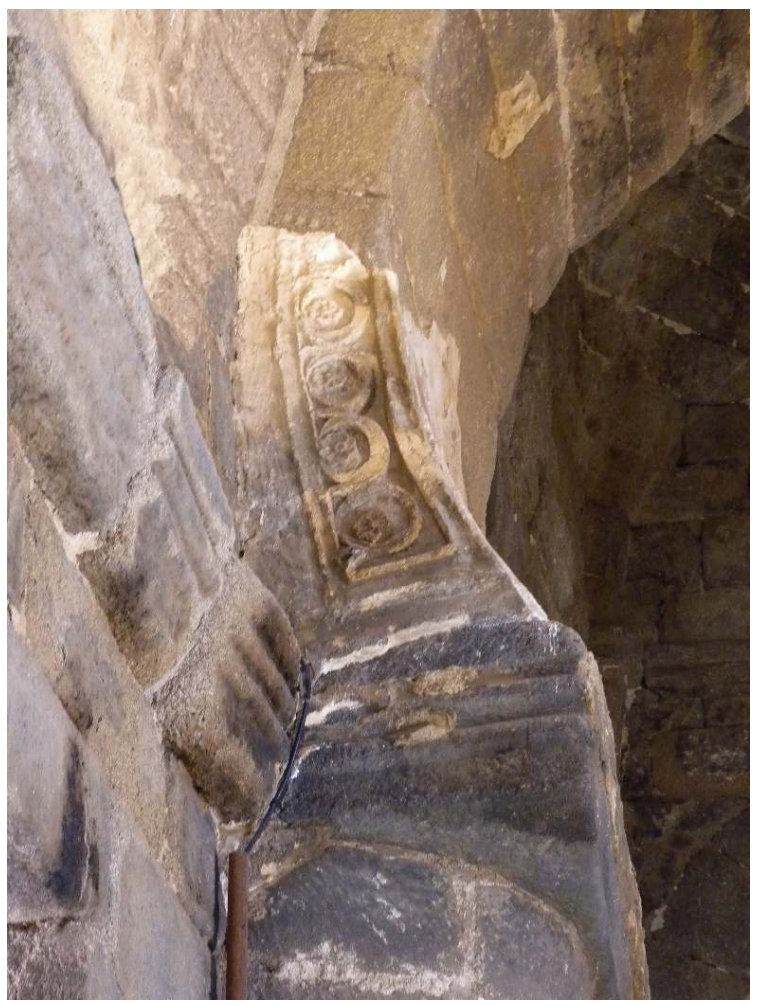

Santa María de Melque (Tolède). Décor sculpté en stuc à rinceau stylisé orné de fleurons à huit pétales.

(C) A. Uscatescu

Fig. 5.

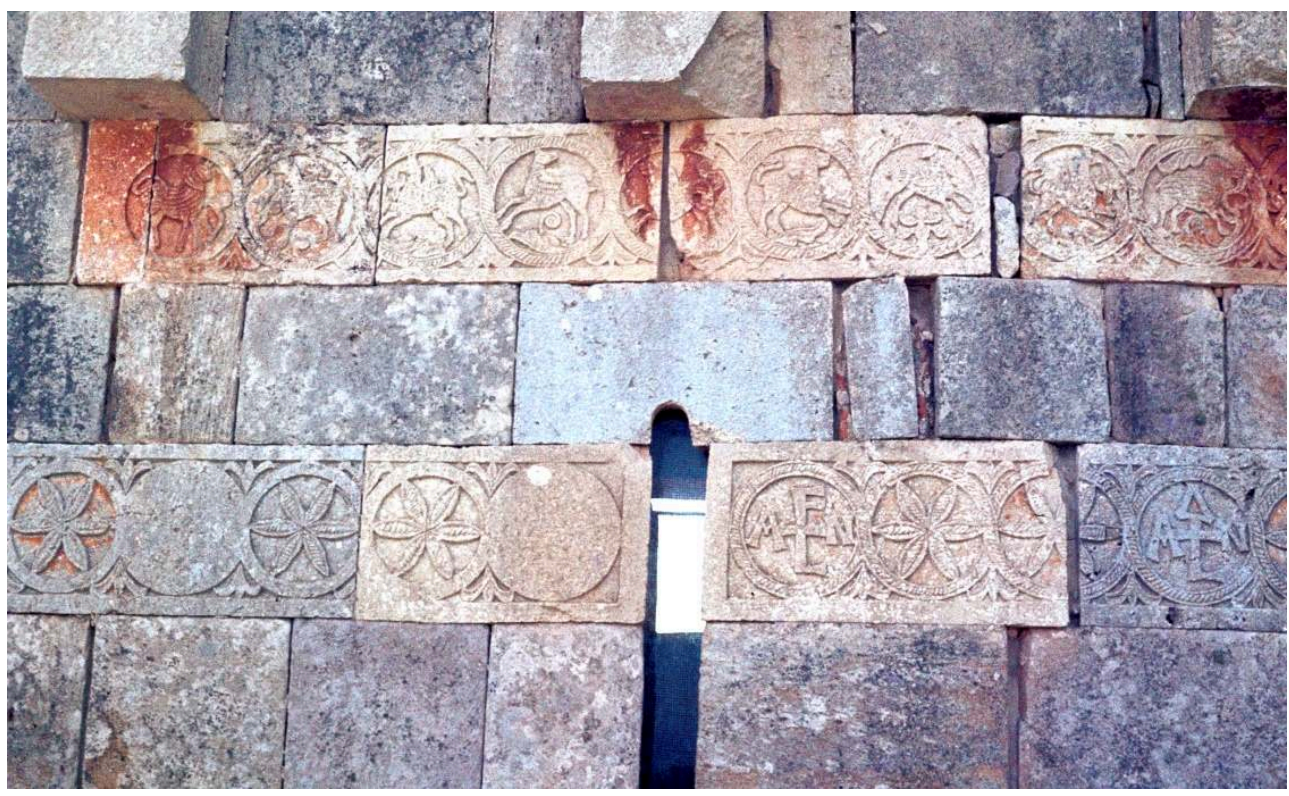

Santa María, Quintanilla de las Viñas (Burgos). Décor sculpté à l'extérieur de l'abside. (c) G. Ripoll

Reste une question de base concernant la sculpture monumentale. Khirbat al-Mafjar est un édifice très classique, aussi classique que sa mosaïque ou que l'art d'époque 
wisigothique. Pourquoi faut-il établir des dépendances entre l'un et l'autre ? Pourquoi ne faut-il pas voir dans les productions - wisigothique et omeyyade - l'Antiquité classique comme une même source d'inspiration, une même tradition ? Comment résoudre les contradictions posées par le matériel lui-même ? Comme, par exemple, quand María Cruz Villalón reprend la question de l'atelier de Mérida ${ }^{50}$. Pour réaffirmer ces hypothèses, Caballero a organisé un colloque sur Visigodos y omeyas traitant seulement la sculpture ${ }^{51}$. Et il y a une autre question, à mon sens capitale : si toute l'architecture, la sculpture et l'épigraphie qui étaient des $\mathrm{VI}^{\mathrm{e}}, \mathrm{VII}^{\mathrm{e}}$ et début $\mathrm{VIII}^{\mathrm{e}}$ siècles ne le sont plus, où sont celles qui correspondent à cette chronologie ? (fig. 6).

Fig. 6

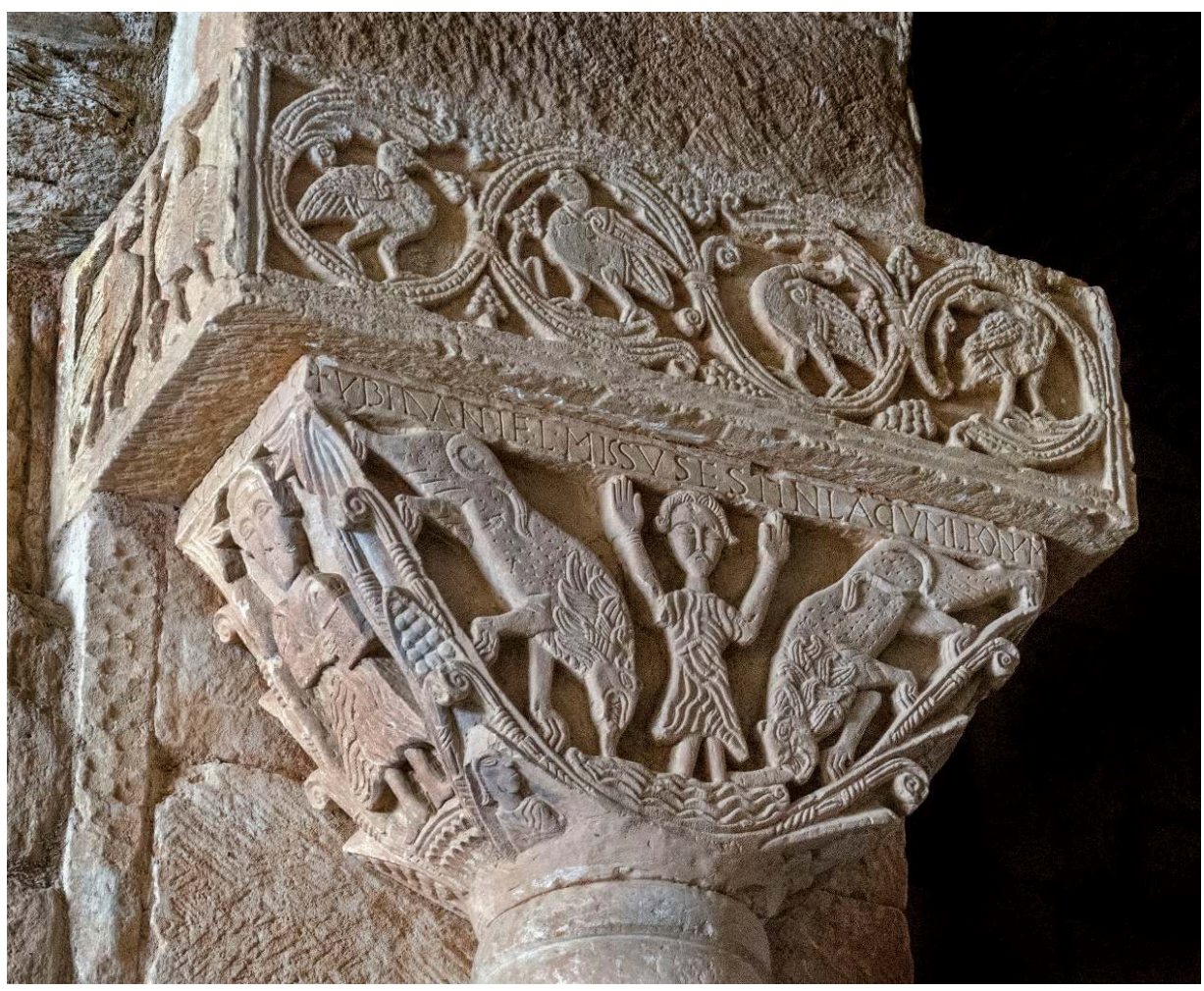

San Pedro de la Nave (Zamora). Chapiteau décoré de la scène de Daniel dans la fosse aux lions, avec l'inscription + ubi Daniel missus est in lacum leonum.

(c) Á. M. Felicísimo

31 Il est vrai que les hypothèses de Caballero sont devenues certitudes pour certains chercheurs $^{52}$, mais il y a d'autres voix qui réclament des relectures attentives afin de privilégier les données fournies par le matériel en soi. Nous l'avons exprimé, il y a déjà plus de vingt ans, dans les « conclusions » du premier colloque, Visigodos y omeyas, en $2000^{53}$. Il en va de même pour Achim Arbeiter ${ }^{54}$ et pour les travaux, à mon sens d'une grande rigueur, de Fedor Schlimbach, concernant tout spécialement San Juan de Baños et Quintanilla de las Viñas ${ }^{55}$, qui se joignent à maints chercheurs venant de toutes les disciplines : l'histoire de l'art ${ }^{56}$, l'architecture ${ }^{57}$, l'architecture et l'épigraphie ${ }^{58}$, l'épigraphie en elle-même ${ }^{59}$, la liturgie ${ }^{60}$, les questions commerciales et économiques ${ }^{61}$. Alexandra Uscatescu et Juan Carlos Ruiz Souza parcourent pas à pas tous les éléments mis en discussion, en facilitant d'autres mécanismes de réponse et d'autres voies de 
compréhension, qui ne soient pas réductionnistes, bien au contraire, et leurs nombreuses observations concordantes mènent à de nouvelles interprétations ${ }^{62}$.

Les premières années du XXI ${ }^{e}$ siècle voient apparaître de nouveaux apports dans le domaine de la sculpture, et je vais en souligner quelques-uns en raison de leur importance, indépendamment des postulats chronologiques qui peuvent être discutés, comme je l'ai signalé auparavant.

En dépit des controverses exposées ci-dessus, le début du XXI ${ }^{e}$ siècle est une période de contributions significatives dans le domaine de la sculpture, ainsi le répertoire d'architecture d'Estrémadure, publié en 2003, qui intègre la sculpture ${ }^{63}$, suivi, quelques années plus tard, par le colloque de la série Visigodos y omeyas portant sur la sculpture ${ }^{64}$, déjà cité précédemment. On doit citer également la thèse de Sergio Vidal, un catalogue raisonné de toute la sculpture figurée, édité luxueusement en $2005^{65}$; le recueil des sculptures de Tolède et celui de Cordoue ${ }^{66}$, qui comporte des productions remarquables (fig. 7) ; la thèse de Javier A. Domingo à propos des chapiteaux, parue en 2011 et suivie par toute une série de publications ${ }^{67}$. Ce matériel, celui des chapiteaux, est de grande importance pour comprendre l'architecture elle-même, et s'y ajoute maintenant l'étude d'une centaine d'exemplaires de Tolède par Jenny Abura, sans oublier sa thèse de doctorat à propos du matériel sculpté de Segobriga déjà citée ${ }^{68}$. Il faut ajouter le Repertorium der christlich-antiken Sarkophage IV, concernant les sarcophages hispaniques, très récemment publié ${ }^{69}$ et le tout aussi récent recueil des sculptures de la région de Badajoz, ancienne Lusitanie ${ }^{70}$. Enfin, vient juste de paraître un volume très attendu à propos des spolia, qui ne résout pas le problème lui-même mais ouvre des nombreuses voies de recherche (fig. 8) ${ }^{71}$. 
Fig. 7

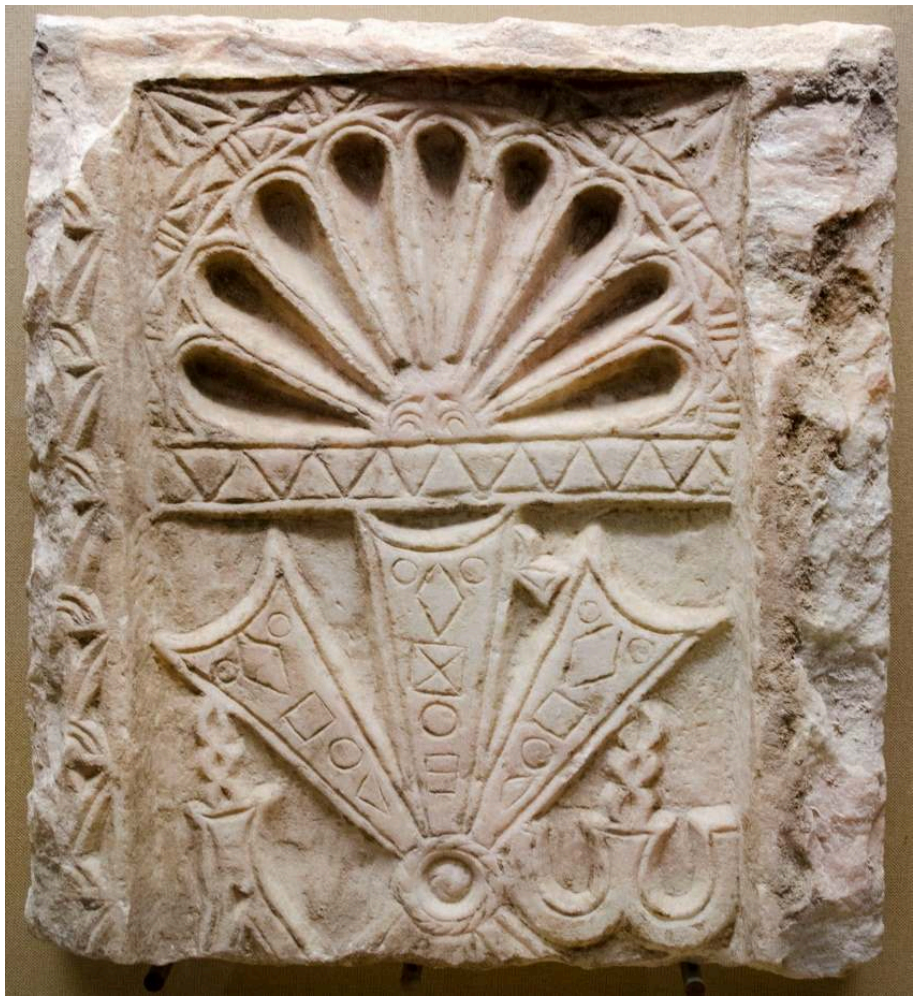

Plaque-niche à coquille et double croix gemmée avec alpha et omega (Museo Visigodo de San Vicente, Mezquita de Córdoba, Cordoue).

(c) Á. M. Felicísimo 
Fig. 8

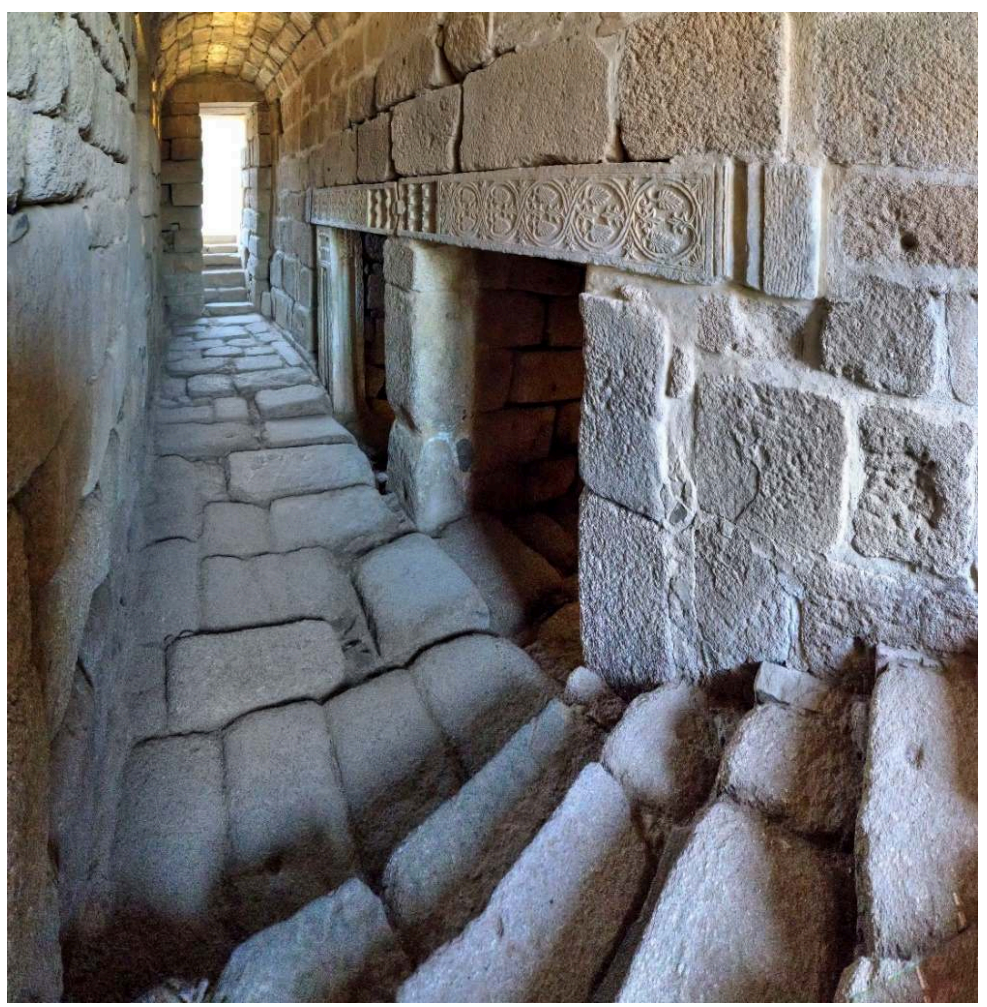

Piliers remployés dans le réservoir d'eaux (aljibe) de la fortification arabe de Mérida, construite au IX siècle (Alcazaba de Mérida, Estrémadure).

(C) Á. M. Felicísimo

Ces contributions - j'ai signalé seulement celles que je considère comme essentielles, car il en existe un très grand nombre que l'on ne peut toutes aborder dans cette intervention - sont des pas de géant, sans le moindre doute, mais des questions de grande importance continuent de se poser lorsque l'on travaille sur la sculpture hispanique : je me réfère tout spécialement aux systèmes d'enregistrement numérique, à la représentation graphique, aux analyses archéométriques et, enfin et surtout, aux problèmes de la provenance des matériaux, aux ateliers et à la chronologie des productions.

\section{Les défis de la recherche}

Compte tenu de ce qui a été exposé précédemment et de la progression de la recherche, il est urgent de trouver des solutions pour cette recherche, mais aussi pour sa diffusion. Une issue possible peut être trouvée dans le « monde globalisé ». Les TIC (Technologies de l'Information et de la Communication), les bases de données et les systèmes informatiques reposant sur l'infrastructure des données spatiales nous conduisent aux corpora numériques sans oublier à aucun moment que les Digital Humanities sont une méthode, mais pas une fin en soi $^{72}$.

Suivent ci-dessous quelques réflexions sur les problèmes que posent, selon nous, les corpora numériques, les implications de la représentation graphique, de la 
photogrammétrie et de la modélisation 3D et, enfin, les analyses archéométriques pour circonscrire les questions de provenance, d'ateliers de production et de chronologie.

L'idée de créer un corpus numérique (en espagnol on utilise l'adjectif « digital ») pour la sculpture va de pair avec le projet CARE/Corpus Architecturae Religiosae Europae (saec. IV$X)$ et sa partie hispanique, CARE-Hispania ${ }^{73}$, en intégrant l'architecture, mais aussi la sculpture et l'épigraphie. Ce projet fonctionne à l'échelle internationale et a homogénéisé un modèle de fiche, proposé par l'équipe menée par M. Jurković et G.P. Brogiolo, plus ou moins adapté aux caractéristiques architectoniques de chaque pays qui y participe ${ }^{74}$.

Pour l'Espagne, nous y travaillons depuis quelques années ${ }^{75}$ et nous avons testé différentes méthodes d'enregistrement. Je veux parler d'un « enregistrement » dans le sens classique : recueillir toutes les informations, textuelles, historiographiques, bibliographiques et graphiques (photos, plans...) essentielles en tant que matériel de base pour rédiger une fiche qui pourrait être éditée en format papier ou consultable dans une plateforme en ligne. Le travail le plus avancé est celui de la Catalogne et des Baléares ${ }^{76}$, qui nous a procuré de grandes surprises, vu le nombre final d'édifices à inventorier : 1319 au total, dont 751 connus par les sources textuelles, tout spécialement des actes de consécration, 314 monuments conservés et 254 mentions épigraphiques, ce qui nous a obligés à expérimenter, à tester différentes méthodes de travail et d'enregistrement et nous a conduits à une réflexion profonde sur le sens et les possibilités du corpus.

Nous n'avons pas suivi la proposition française de CARE, qui a créé une plateforme " wikicare ", grâce au travail tenace d'une équipe pilotée par Pascale Chevalier ${ }^{77}$. Nous avons testé d'autres possibilités en utilisant des plateformes déjà existantes pour ne pas doubler le travail. Nous nous sommes focalisés sur l'épigraphie concernant l'architecture ou le mobilier liturgique de la Catalogne. C'est-à-dire que nous avons fait le travail de base, récolter toutes les inscriptions : les différentes éditions, transcriptions, interprétations, etc., ainsi que les pdf de tous les textes et les photographies, ce qui a représenté un très long travail, pour un matériel qui n'est pas facile. On a réuni ainsi 254 inscriptions datées entre le $\mathrm{IV}^{\mathrm{e}}$ et le $\mathrm{XI}^{\mathrm{e}}$ siècle (fig. 9).

Fig. 9

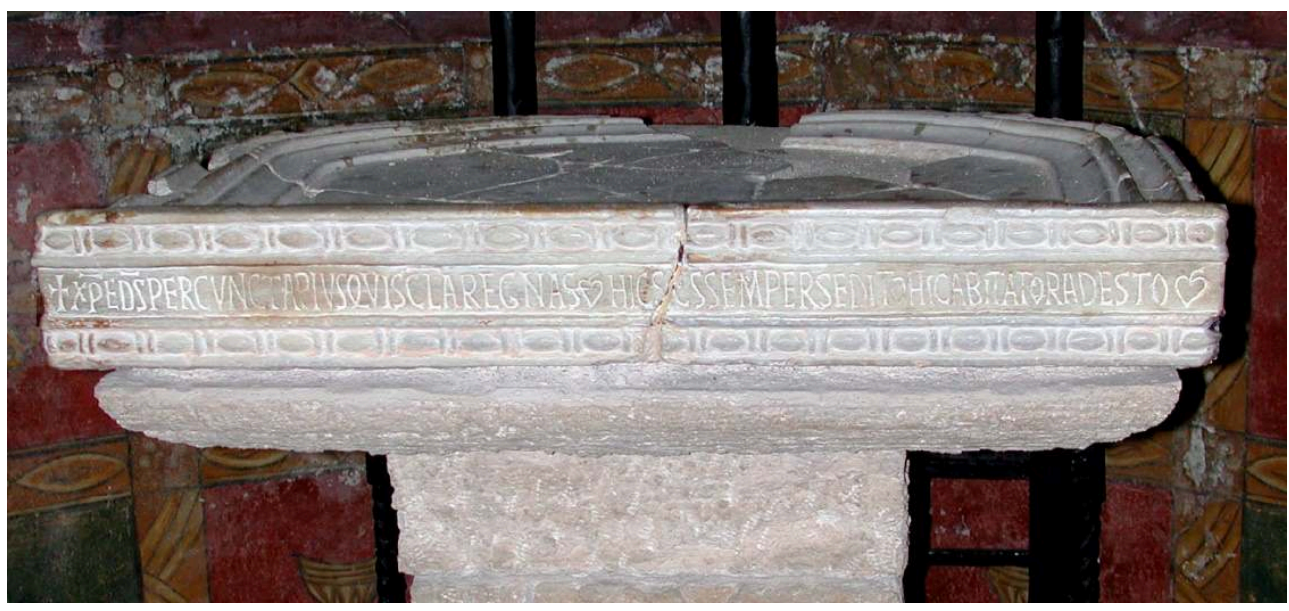

Table d'autel avec inscription de Sant Feliuet de Vilamilanys (Sant Quirze del Vallès, Rubí, Barcelone) (ICERV 557) (d'après D. Rico, 2009, fig. 6, op. cit. note 58). 
Nous nous sommes mis d'accord avec Isabel Velázquez de l'Université Complutense de Madrid, qui dirige l'archive épigraphique AEHTAM/Archivo Epigráfico de Hispania Tardoantigua y Medieval (Universidad Complutense) ${ }^{78}$, pour introduire tout ce matériel dans sa plateforme et il est déjà consultable en ligne. Mais l'AEHTAM est un simple recueil des donnés, pas une banque de données ; il n'intègre pas tous les éléments documentaires fournis, à l'exception d'une ou deux photographies. Cette archive/ recueil AETHAM est donc très loin d'un modèle comme celui proposé par le Corpus of Anglo-Saxon Stone Sculpture ${ }^{79}$, pour citer un exemple portant sur la sculpture, qui permet de consulter tous les documents en ligne et de localiser les sites sur une carte associée.

41 Il nous faudrait donc trouver la méthode la plus adéquate pour travailler dans le sens des plateformes numériques les meilleures pour la recherche, en donnant la possibilité de consultation, mais aussi de collaboration. Les longues discussions se sont concrétisées sur le choix d'un système informatique IDS (Infrastructure de Données Spatiales), conçu, en premier lieu, pour l'épigraphie, EPIARQ, par Rocío Gutiérrez, Mercedes Farjas et Isabel Velázquez ${ }^{80}$. Il s'agit d'une standardisation de la méthodologie de catalogage et $d$ 'analyse spatiale, selon la directive européenne INSPIRE, les standards OGC et les normes ISO 19100, pour créer une Infrastructure de Données Spatiales (IDS). L'objectif principal est de pouvoir intégrer tous les types de bases de données (traditionnelles ou numériques) dans un cadre interopérable qui permet d'étudier, par une analyse historique et comparative, l'évolution des inscriptions à l'aide des outils de géovisualisation / SIG.

Pour CARE-Hispania nous avons travaillé dans ce même sens, établissant la méthodologie et en intégrant tous les éléments d'architecture, sculpture, mobilier liturgique, épigraphie... : CARE_SHDI Infraestructura de Datos Espaciales Históricos para Arqueología, Arquitectura y Patrimonio Cultural (Infrastructure de Données Spatiales pour l'Archéologie, l'Architecture et le Patrimoine Culturel) ${ }^{81}$. La proposition de créer une infrastructure de données spatiales résulte de la réflexion sur les problèmes posés par l'analyse de l'architecture et du patrimoine bâtis entre le $\mathrm{IV}^{\mathrm{e}}$ et le XI $\mathrm{XI}^{\mathrm{e}}$ siècles, dans le cadre du projet CARE-Hispania lui-même. La méthode centrée sur l'individualité de chaque édifice, oubliant qu'il fait partie d'un tout en constante transformation, nécessite de trouver de nouveaux systèmes de recherche et de diffusion du patrimoine en général et du patrimoine architectural, archéologique et religieux en particulier. Nous pensons qu'il est nécessaire de développer une SHDI, infrastructure de données spatiales historiques, capable d'intégrer, de stocker, d'éditer, d'analyser, de partager et d'afficher toutes les informations géographiquement référencées et structurées de façon temporelle, capable de dessiner le temps, c'est-à-dire des réalités historiques dont la portée est difficile à prévoir. Le défi de CARE_SHDI est de devenir une entité formelle et durable qui intègre les différents agents du domaine du patrimoine culturel, avec la ferme conviction que cela signifie une avancée vers un nouvel espace d'informations interconnectées au service de la société. L'expérience de nos projets nous montre l'extrême nécessité d'établir des synergies et de créer des actions et des mécanismes efficaces pour l'innovation avec de nouveaux systèmes de gestion du patrimoine et du numérique.

43 Les corpora numériques doivent être accompagnées de la représentation graphique qui, aujourd'hui, doit se faire par photogrammétrie et modélisation 3D $\mathrm{D}^{82}$. C'est un défi non négligeable si l'on veut arriver à intégrer l'ensemble des données. Et il faut être attentif à différencier la sculpture hors contexte, en tant qu'objet d'art pouvant offrir des 
résultats importants, surtout si elle est accompagnée d'inscriptions (fig. 10), de la sculpture en contexte spatio-temporel, qui est donc en relation avec une stratigraphie qui permet de connaître la séquence chronologique et l'évolution d'une construction.

Fig. 10

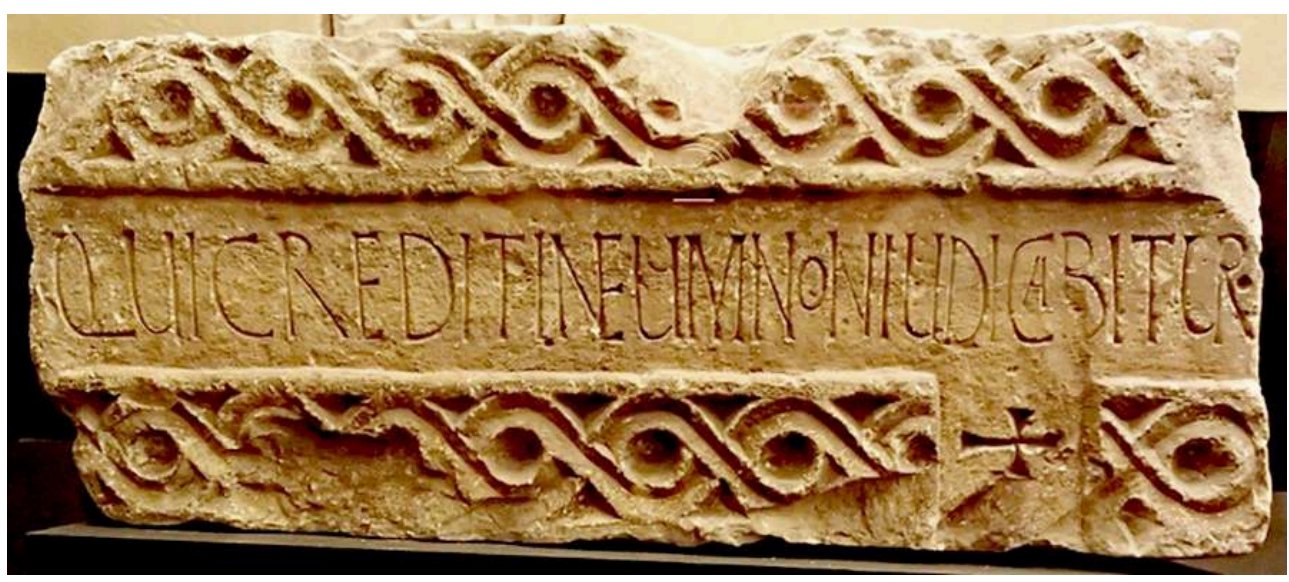

Fragment d'inscription avec le texte du Credo : qui credit in eum non iudicabitur ; encadrée par motifs en tresse (ICERV 554, CIL II2/13, 63, texte de I. Velázquez), provenant des environs de l'église de SainteLéocadie, extra muros de Tolède (Museo de los Concilios y de la Cultura Visigoda, Tolède).

(c) Asociación de Amigos del Arte Altomedieval Español / 4AsE permettant la modélisation et donc l'étude optimale de la sculpture et l'analyse de l'édifice. La sculpture, en tant que partie essentielle de l'espace, est dotée d'un volume, qui, grâce aux nouvelles méthodes de représentation (sans mépriser les techniques traditionnelles et académiques), peut être offert en vision intégrale et dynamique à 360 degrés, métrique et géoréférencée. Le produit final est essentiel pour la recherche, mais aussi pour la diffusion de cette recherche. d'intégration de toutes les données pour une correcte reconstruction historique, il faut affronter sérieusement les problèmes intrinsèques à l'œuvre sculptée - les productions des ateliers, la provenance des matériaux et la chronologie - en utilisant l'archéométrie et toutes les méthodes de datation convenables. C'est, très probablement, un des défis les plus importants ${ }^{83}$. Et, si on veut faire un travail sérieux, il nous faudrait individualiser les problèmes de chaque contexte, oublier les généralités et établir systématiquement des analyses archéométriques micro et macrorégionales pour obtenir une bonne mise en série et, en définitive, une séquence chronologique plus proche du matériel et de son contexte. La caractérisation et l'identification des provenances et des ateliers, fixes ou itinérants, sont fondamentales pour mieux cerner les systèmes d'achat et de vente en fonction des centres de production connus et des marchés à courte et longue distance, afin de faciliter la compréhension de leur importance en tant qu'élément des structures économiques et commerciales. Cela permettrait d'identifier les traits déterminants des différents ateliers, en laissant de côté les questions de style et de typologie, tout en procurant les bases nécessaires pour établir une chronologie.

46 L'étude des provenances du matériel est essentielle et de notables progrès ont été réalisés concernant les marbres qui témoignent des contacts commerciaux entre 
l'Orient et l'Occident, ce qui est largement attesté par les céramiques et les épaves de navires comme celle de Marzamemi ${ }^{84}$ (à l'extrémité sud de la côte syracusaine, non loin de Noto) et le site de Favaritx ${ }^{85}$ (au nord-est de Minorque, sur le cap du même nom, battu par des vents violents), avec la cargaison de nombreux objets métalliques, dont ceux à caractère liturgique, sans doute destinés à un édifice ecclésiastique - pour citer seulement deux des meilleurs exemples.

Les observations à l'œil nu doivent nécessairement être vérifiées par les analyses archéométriques. Des exemples de cette démarche offrent des résultats de grande importance et donc la nécessité de faire de bonnes mises en série archéométriques en vue de mieux discerner les provenances et la chronologie. Je propose seulement quelques cas, à mon sens très pertinents.

Un dossier exemplaire, qui corrobore une fois de plus l'existence des routes commerciales entre l'Orient et l'Occident, comme nous venons de le mentionner, est celui des carrières de Göpteke qui ont approvisionné les nombreux ateliers de sculpture d'Aphrodisias au moins jusqu'au $\mathrm{VI}^{\mathrm{e}}$ siècle ${ }^{86}$. Les produits d'Aphrodisias atteignaient l'Hispanie, comme l'a conjecturé Javier Arce à propos du marché (nundina), de Valdetorres del Jarama (au nord de Madrid) (fig. 11) où se vendaient des produits orientaux, des ivoires et des sculptures, notamment en marbre nero antico de Göpteke, acheté selon toute probabilité à un marchand qui portait les matériaux depuis un des ports de la côte de Carie en Asie mineure ${ }^{87}$.

Fig. 11

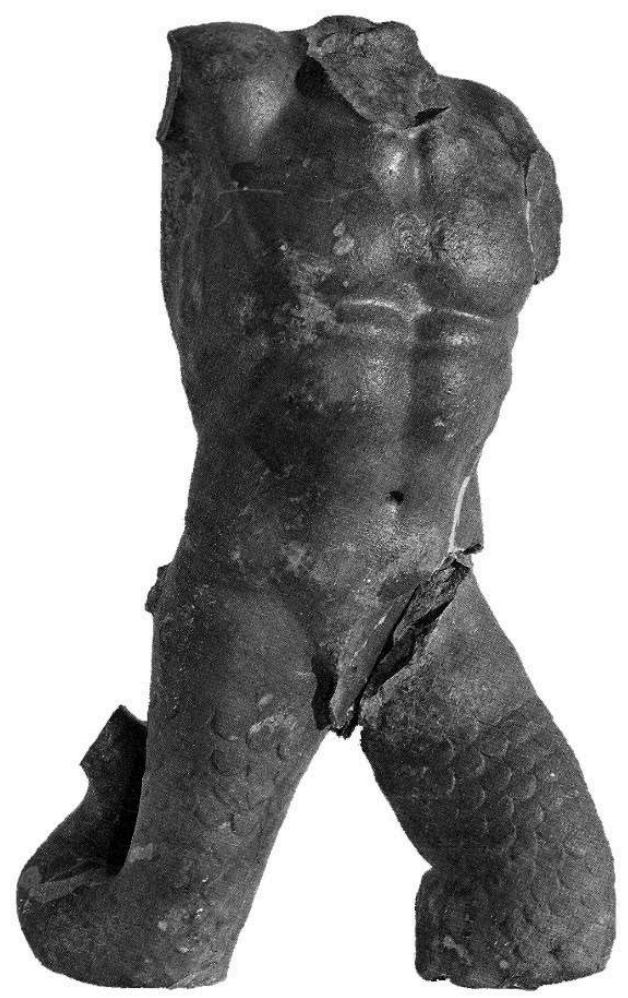

Sculpture de triton provenant de Valdetorres de Jarama (Madrid) en marbre nero antico de Göpteke (Aphrodisias, Carie, Turquie actuelle), (d'après J. Arce, L. Caballero et M.Á. Elvira, 1997, op. cit. note 87).

Il faut citer deux importants volumes dédiés à l'utilisation du marbre en Hispanie, qui révèlent l'importance des extractions de l'anticlinal d'Estremoz au Portugal, l'ancienne 
Lusitanie, en procurant du matériel de taille à toute la péninsule ${ }^{88}$. Les différentes analyses pratiquées sur divers objets sculptés montrent l'importance des carrières dans l'anticlinal d'Estremoz, à mi-chemin entre Evora et Mérida ${ }^{89}$. C'est l'une de ces exploitations qu'on connaissait bien et il était clair qu'une grande partie, sinon toutes, des productions lusitaines venaient d'Estremoz, mais cela va plus loin, puisque commence à être attestée l'exportation de ce marbre vers d'autres régions.

50 Je donne ici l'exemple d'un sarcophage bien connu, celui d'Ithacius à Oviedo (fig. 12), dans les Asturies, qui a toujours été considéré comme une production aquitaine. Finalement, l'étude et les analyses menées par S. Vidal et V. García-Entero montrent que le marbre est de provenance lusitaine ${ }^{90}$.

Fig. 12
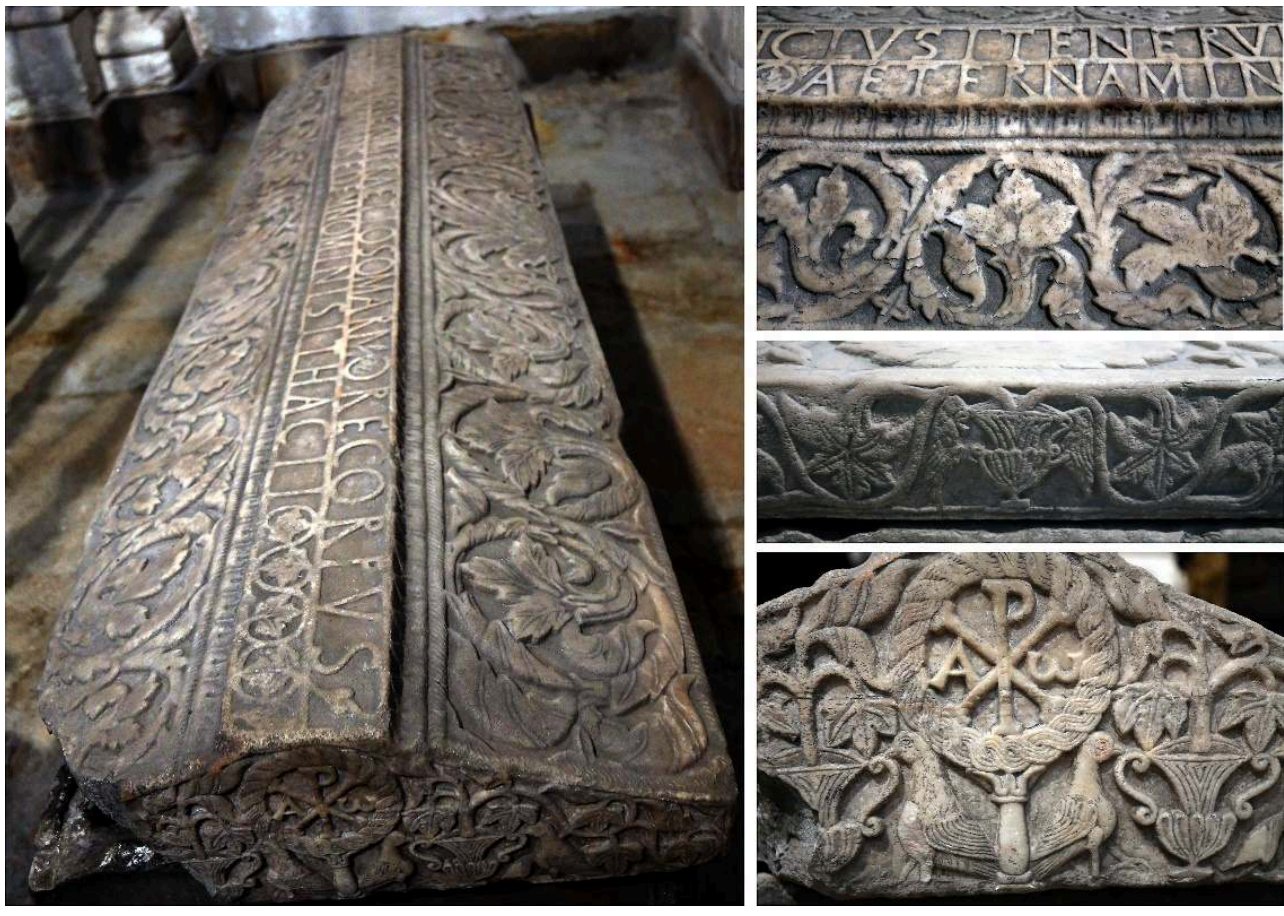

Sarcophage d'Ithacius (Santa María, Oviedo, Asturies).

(C) B. Torre Miguel

51 Ce n'est pas un cas unique, bien au contraire, les exemples peuvent être multipliés, comme ceux des sarcophages du mausolée de Vegas de Pueblanueva à Tolède ${ }^{91}$, du couvercle de sarcophage avec la scène du cycle de Jonas de Carranque, également à Tolède ${ }^{92}$, et du sarcophage de Tui de Pontevedra, en Galice ${ }^{93}$, parmi d'autres.

Parmi les analyses de provenances, il convient de mentionner certains sarcophages de la nécropole paléochrétienne de Tarragone. Des analyses pétrographiques effectuées il y a quelque temps ont mis fin à la discussion qui préoccupait tant Helmut Schlunk et Pere de Palol au sujet de l'étroite ressemblance entre les sarcophages à décor de strigiles de Tarraco et ceux de la région de Carthage. Certains de ces spécimens de Tarraco sont taillés dans la pierre « kadhel » provenant des carrières situées au sud de Carthage (Djebel Kadhel, Borj Cedria) ${ }^{94}$.

53 Le sarcophage lisse de Tui, cité précédemment, ouvre une question qui est, à mon avis, fondamentale. L'accent des recherches mis sur les ateliers locaux, mais toujours sur les 
sarcophages sculptés (avec une décoration figurative, géométrique et/ou végétale) ${ }^{95}$, montre qu'il n'existe pas d'études approfondies sur les ateliers locaux qui produisent d'autres types de sarcophages, ceux qui sont lisses, sans aucune sorte de décoration, parmi lesquels il faut inclure ceux de la période romaine classique, également lisses, souvent retravaillés et réutilisés pendant l'Antiquité tardive et le haut Moyen Âge. Il reste encore des recherches sérieuses à mener sur ce type de sarcophage et sur les différents contextes urbains et ruraux de toute cette réalité matérielle, comme ceux de Mérida ou bien la presque centaine d'exemples de la nécropole paléochrétienne de Tarragone, en grand partie taillés en calcaire local de Santa Tecla ${ }^{96}$ (fig. 13). Les résultats contribueront à améliorer la connaissance des commanditaires, des différences sociales, du réseau des marchés et des paramètres économiques et religieux. Le problème va au-delà des $\mathrm{IV}^{\mathrm{e}}$ et $\mathrm{V}^{\mathrm{e}}$ siècles, car que faire de tous ces sarcophages, dont le nombre n'est pas négligeable, qui apparaissent dans les nécropoles dites wisigothiques du plateau castillan, comme Duratón ou Madrona ${ }^{97}$ ? Et une autre question fondamentale, en ce qui concerne la sculpture funéraire, est celle des épitaphes qui contiennent également des éléments décoratifs, sculptés et iconographiques et qui appartiennent - ou non - au même horizon chronologique.

Fig. 13

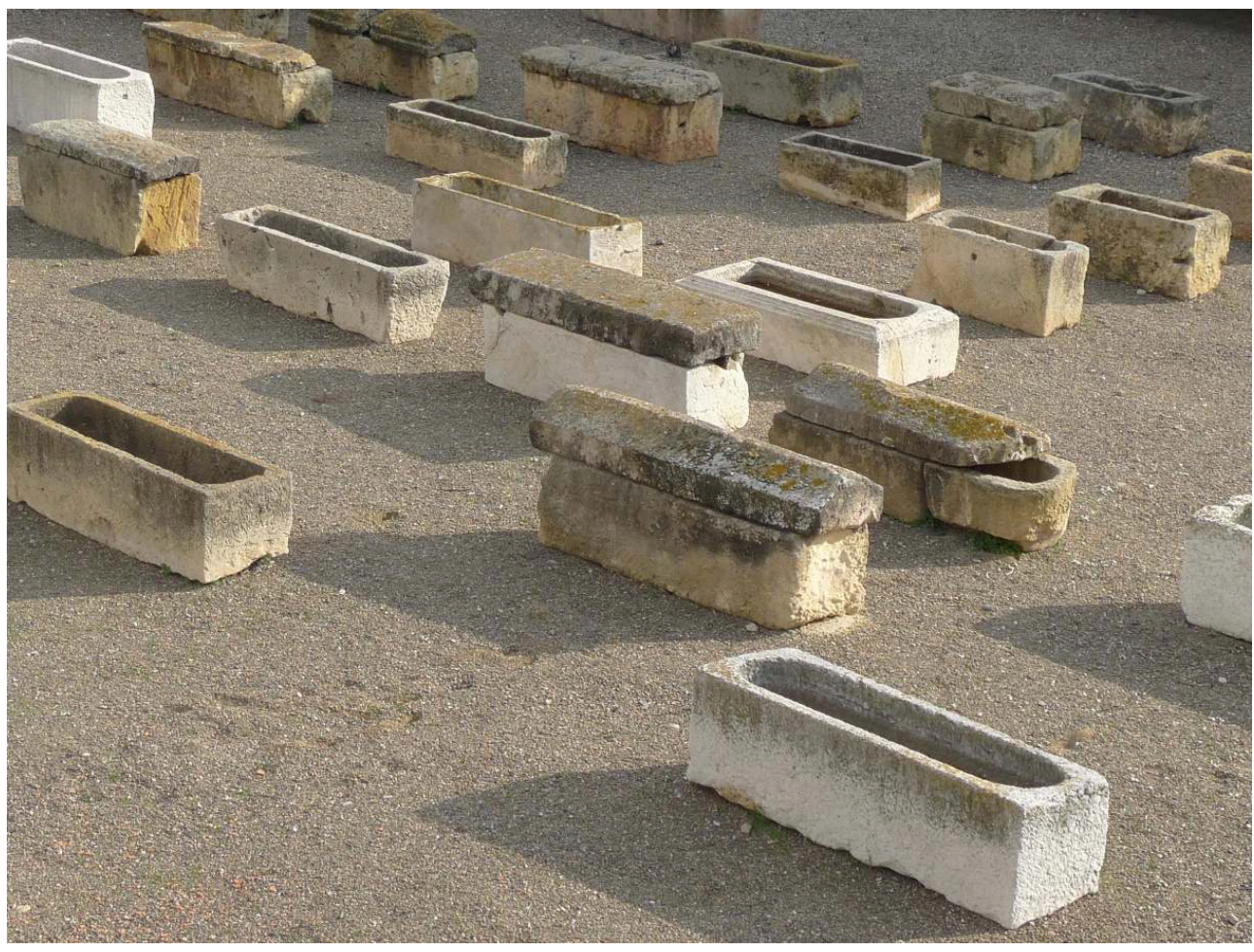

Tarragone, sarcophages lisses de la nécropole paléochrétienne.

(c) G. Ripoll

Last but not least, je tiens tout particulièrement à attirer l'attention sur le projet EMCHAHE/Early Medieval Churches: History, Archaeology and Heritage, réalisé en Galice, qui souligne l'importance de la recherche de nouvelles méthodes d'étude et de l'application de systèmes d'analyse (notamment le ${ }^{14} \mathrm{C}$ et l'OSL/Optically Stimulated Luminescence/Luminescence Optiquement Stimulée pour les mortiers), ainsi que le SIG, sans oublier les sources textuelles, afin d'évaluer l'architecture ecclésiastique 
avant $\mathrm{l}^{\prime}$ an $\mathrm{Mil}^{98}$. Ces travaux multidisciplinaires abordent la sculpture dans son contexte architectural et constituent un excellent exemple méthodologique. À partir de l'étude de l'édifice en lui-même, réalisée sous tous les points de vue possibles, comme nous venons de le dire, la sculpture est intégrée dans la séquence chronostratigraphique, fournissant des résultats de grande pertinence.

Les pages précédentes sont une réflexion sur ces problèmes historiographiques, méthodologiques et de fond. Elles permettent de constater la richesse de la sculpture hispanique et les progrès, mais surtout les difficultés, telles que l'intégration et l'accessibilité de toutes les données, (nécessités urgentes pour une bonne séquence chronologique), auxquelles sont confrontés les chercheurs intéressés par l'architecture et la sculpture de l'Antiquité tardive et du haut Moyen Âge. Les technologies innovantes offrent des possibilités objectives qu'il faut mettre au service de l'investigation de la sculpture pour la caractériser et la contextualiser dans l'espace et dans le temps. Au moment d'achever la rédaction de ce manuscrit, la presse a donné la nouvelle d'une remarquable découverte. Les fouilles dans la villa de Los Villaricos (Mula, Murcie) concernant la nécropole des $\mathrm{VI}^{\mathrm{e}}$ et $\mathrm{VII}^{\mathrm{e}}$ siècles (datations $\mathrm{C}^{14}$ ), qui s'étend sur les structures et autour de la salle de réception transformée, très probablement, en basilique chrétienne, ont mis à découvert un type de sarcophage méconnu dans les productions hispaniques ${ }^{99}$. Il s'agit d'une cuve lisse dotée d'un couvercle monolithe à double pente. Chacun des grands panneaux de celui-ci comporte un décor encadré par un filet double rectiligne, formé d'une tresse à trois brins et onze grands œillets avec feuilles cordiformes dans les écoinçons supérieurs, d'une finition remarquable. Sur l'un des petits côtés du couvercle est figuré un chrisme flanqué par l'w (à gauche) et l'a (à droite), le tout inscrit dans une couronne perlée. Sans vouloir m'avancer sur l'étude de détail et les analyses nécessaires, ce sarcophage est un exemple de la qualité du travail des artisans locaux et de l'emploi de répertoires décoratifs communs à l'Antiquité tardive en Hispanie.

\section{NOTES}

1. Je remercie très vivement Pierre-Yves Le Pogam et Bénédicte Mérel-Brandenburg de m'avoir invitée à participer à ces encourageantes journées d'étude, ainsi que pour leur détermination à résoudre toutes les contraintes pandémiques. Je tiens également à remercier R. Alonso, J. Arce, X. Barral, J. Behaim, E. Carrero et A. Uscatescu pour la lecture attentive et la discussion ; ainsi que les personnes qui m’ont aidée à préparer les illustrations : R. Álvarez, Á. M. Felicísimo, P. GarcíaDiego et l'Asociación de Amigos del Arte Altomedieval Español (4AsE), B. Torre Miguel et A. Uscatescu.

2. Manuel Sotomayor, Datos históricos sobre sarcófagos romano-cristianos de España, Grenade, 1973 et Id., Sarcófagos romano-cristianos de España. Estudio iconográfico, Grenade, 1975.

3. Cornide était accompagné de l'architecte Melchor de Prado y Merino, qui a réalisé les planimétries. Voir José Cornide, « Noticia de las antigüedades de Cabeza de Griego reconocidas de orden de la Real Academia de la Historia por su Académico de número D. Joseph Cornide ", Memorias de la Real Academia de la Historia, III, 1799, pp. 71-244. 
4. Voir la compilation bibliographique réalisée par Juan Manuel Abascal, Martin Almagro Gorbea et Rosario Cebrián dans : https://www.ua.es/personal/juan.abascal/segobriga_biblioteca.html.

5. La thèse de doctorat de Jenny Abura, Architekturdekoration des 4. bis 7. Jhs. in Segobriga (Saelices, Spanien), Georg-August-Universität Göttingen, 2016, sous la direction de Achim Arbeiter, doit être publiée prochainement. Pour consulter les premiers résultats publiés : Jenny Abura, "Neue Forschungsergebnisse $\mathrm{zu}$ den Werkstattbeziehungen der spätantiken Baudekoration aus Segobriga (Saelices, Spanien) », Oppidum - Civitas - Urbs. Städteforschung auf der Iberischen Halbinsel, zwischen Rom und al-Andalus, sous la direction de Sabine Panzram, Berlin, LIT Verlag, Geschichte und Kultur der Iberischen Welt 13, 2017, pp. 493-518.

6. Voir pour les périodes précédentes : Gisela Ripoll, « Historiografía y numismática visigodas entre los siglos XVI y XVIII: Ambrosio de Morales y Enrique Flórez », Les mérovingiens, archéologie et historiographie (Actes des VI ${ }^{\mathrm{e}}$ journées nationales de l'AFAM, 1984), Paris, 1989, pp. 58-64.

7. Juan Bordes, Monumentos Arquitectónicos de España (1852-1881), Calcografía Nacional de la Real Academia de Bellas Artes de San Fernando, Madrid, Universidad Politécnica de Madrid et Escuela Técnica Superior de Arquitectura de Madrid, 2014. Voir la base des données Monumentos Arquitectónicos de España dans la collection de l'Académie Royale des Beaux-Arts : https:// www.academiacolecciones.com/estampas/monumentos-arquitectonicos-espana.php [Date de consultation : 23.07.2021].

8. G. Ripoll et Eduardo Carrero, « Art wisigoth en Hispania : en quête d'une révision nécessaire », Perspective. Revue de l'Institut National d'Histoire de l'Art, 2009/2, pp. 256-276.

9. José Amador de los Ríos, El arte latino-bizantino en España y las coronas visigodas de Guarrazar, Madrid, Memorias de la Real Academia de San Fernando, 1861.

10. Tout en tenant compte du fait qu'il s'agit d'hypothèses qu'il faudra corroborer avec des interventions archéologiques : Juan Manuel Rojas Rodríguez-Malo, Alejandro Vicente Navarro et Christoph Eger, «La basílica de Guarrazar desde los hallazgos arqueológicos », dans X Jornadas Visigodas Guadamur 2017, Tolède, Ayuntamiento de Guadamur, 2017, pp. 59-86.

11. G. Ripoll, « Il Tesoro di Guarrazar. La tradizione dell'oreficeria nella tarda antichità », dans Tesori. Forme di accumulazione della ricchezza nell'alto medioevo (secoli V-XI), sous la direction de Sauro Gelichi et Cristina La Rocca, Rome, Viella Editrice, 2004, pp. 207-240. Et le volume avec plusieurs contributions : Alicia Perea (ed.), El tesoro visigodo de Guarrazar, Museo Arqueológico Nacional, Madrid, 2001.

12. http://biblioteca.cchs.csic.es/digitalizacion_tnt/index.html, voir El Catálogo Monumental de España (1900-1961). Investigación, restauración y difusión, Madrid, Ministerio de Cultura, 2012. [Date de consultation : 23.09.2021].

13. Josep Puig i Cadafalch, Antoni De Falguera et Josep Goday, L'Arquitectura romànica a Catalunya, 3 vols., Barcelone, Institut d'Estudis Catalans, 1909-1918. J. Puig i Cadafalch, Escrits d'arquitectura, art $i$ política, sélection, introduction et édition de Xavier Barral i Altet, Barcelone, Institut d'Estudis Catalans, Memòries de la Secció Històrico-Arqueològica, 62, 2003. Josep Puig i Cadafalch, arquitecte de Catalunya (1867-1956), Recull de les actes del Congrés celebrat els dies 18-21 d'octubre de 2017 $i$ altres escrits dedicats, sous la direction de Mireia Freixa et Eduard Riu-Barrera, Barcelone, Edició commemorativa de l'Any Puig i Cadafalch 2017-Departament de Cultura de la Generalitat de Catalunya, 2021.

14. I. Precedents: L'arquitectura romana. L'arquitectura cristiana preromànica, 1909. II. L'arquitectura romànica fins a les darreries del segle XI, 1911.Voir notamment la réédition du volume I, avec le commentaire et la mise à jour des différents chapitres : L'Arquitectura cristiana preromànica $a$ Catalunya de J. Puig i Cadafalch, A. de Falguera i J. Goday. Edició facsímil i textos d'actualització, sous la direction de Josep Guitart, Montserrat Pagès, Isabel Rodà et Josep Maria Sans i Travé, Barcelone, IEC-ANC-MNAC-ICAC, 2016. 
15. María Cruz Villalón, «El paso de la Antigüedad a la Edad Media. La incierta identidad del arte visigodo ", Arte de épocas inciertas: De la Edad Media a la Edad Contemporánea, Saragosse, 2009, pp. 7-45 (voir pp. 8-11).

16. J. Puig i Cadafalch, L'Art wisigothique et ses survivances. Recherches sur les origines et le développement de l'art en France et en Espagne du IV ${ }^{e}$ au XII ${ }^{e}$ siècle, Paris, F. de Nobele, 1961.

17. Francis Salet, «Puig i Cadafalch (J.), L'art wisigothique et ses survivances. Recherches sur les origines et le développement de l'art en France et en Espagne du IV $V^{e}$ au XII ${ }^{e}$ siècle, 1961 » (compte-rendu), Bulletin monumental, t. 120, 1962, $\mathrm{n}^{\circ}$ 4, pp. 408-410 (voir p. 408).

18. Emilio Camps Cazorla, «El arte hispanovisigodo », Historia de España, sous la direction de Ramón Menéndez Pidal, vol. III, Madrid, 1940, pp. 491-666.

19. Helmut Schlunk, Die Ornamentik in Spanien zur Zeit der Herrschaft der Westgoten, Berlin, 1930-1936. Id., « El arte decorativo visigodo », Boletín Bibliográfico, 3, 1944, pp. 14-35.

20. H. Schlunk, "Relaciones entre la Península Ibérica y Bizancio durante la época visigoda », Archivo Español de Arqueología, XVIII, 1945, pp. 177-204 ; sujet sur lequel il est revenu des années plus tard : Id., "Byzantinische Bauplastik aus Spanien ", Madrider Mitteilungen, 5, 1964, pp. 234-254. Voir l'analyse critique du byzantinisme de Helmut Schlunk dans Jean-Marie Hoppe, « La sculpture wisigothique et le monde byzantin », Byzantiaka, 110, 1991, pp. 61-95. Sujet repris récemment par Fabrizio Sanna, « Aportaciones estilísticas y formales de filiación bizantina en la escultura visigoda del Sureste peninsular: los casos de Algezares (Murcia), Begastri (Cehegín) y Alcudia de Elche (Elche) », RiMe Rivista dell'Istituto di Storia dell'Europa Mediterranea 7.III, 2020, pp. 31-110.

21. H. Schlunk, «Arte visigodo » et «Arte asturiano », dans Blas Taracena Aguirre, Pere Batlle Huguet et Helmut Schlunk, Ars Hispaniae, vol. II, Madrid, Plus Ultra, 1947, pp. 225-416.

22. H. Schlunk et Theodor Hauschild, Die Denkmäler der frühchristlichen und westgotischen Zeit, Mayence, Hispania Antiqua, 1978.

23. H. Schlunk, "Beiträge zur kunstgeschichtlichen Stellung Toledos im 7. Jahrhundert », Madrider Mitteilungen, 11, 1970, pp. 161-186. Id., " Entwicklungsläufe der Skulptur auf der Iberischen Halbinsel vom 8. bis 11. Jahrhundert ", Kolloquium über Spätantike und Frühmittelalterliche Skulptur, III, Mayence, 1974, pp. 121-138.

24. Pere de Palol, «Esencia del arte hispánico de época visigoda : romanismo y germanismo », I goti in Occidente, problemi, Settimane di Studio del Centro Italiano di Studi sull'Alto Medioevo, Spolète, 1956, pp. 65-126.

25. P. de Palol, Arqueología cristiana de la España romana, siglos IV-VI, Madrid-Valladolid, 1967.

26. Je ne donne comme exemple que deux textes qui concernent directement le sujet de ce travail : Id., Tarraco hispanovisigoda, Tarragone, 1953 ; Id., « Placas en cerámica decoradas, paleocristianas y visigodas ", Scritti di storia dell'Arte in onore di Mario Salmi, Rome, 1961, pp. 131-154, parmi d'autres publications tout aussi remarquables, comme celles sur les sarcophages et les autels.

27. P. de Palol, Arte hispánico de la época visigoda et Arte paleocristiano en España, Barcelone, Ediciones Polígrafa, 1968 et 1969.

28. P. de Palol, « Arte y Arqueología », Historia de España Menéndez Pidal, sous la direction de José María Jover Zamora, vol. III. 2, España visigoda. La Monarquía. La Cultura. Las Artes, Madrid, Espasa Calpe, 1991, pp. 269-428.

29. Del romà al romànic. Història, art $i$ cultura de la Tarraconense mediterrània entre els segles IV $i \mathrm{X}$, sous la direction de P. de Palol et Antoni Pladevall, Barcelone, Enciclopèdia Catalana, 1999. Pour toute la série de la Catalunya Romànica, voir infra note 34.

30. José Vives, Tomás Marín et Gonzalo Martínez, Concilios visigóticos e hispano-romanos, BarceloneMadrid, CSIC, 1963. José Vives, Inscripciones cristianas de la España romana y visigoda, Barcelone, 1969 (=ICERV).

31. Thilo Ulbert, Studien zur dekorativen Reliefplastik des östlichen Mittelmeerraumes. Schrankenplatten des 4.-10. Jahrhunderts, Munich, Miscellanea Byzantina Monacensia, 10, 1969, suivi par Id., " 
Skulptur in Spanien (6.-8. Jh.) », Kolloquium über Spätantike und Frühmittelalterliche Skulptur, 2, Mayence, 1970, pp. 25-34.

32. Je voudrais rappeler ici le texte signé conjointement par Noël Duval et Paul-Albert Février, « Le décor des monuments chrétiens d'Afrique (Algérie, Tunisie) », Actas del VIII Congreso Internacional de Arqueología Cristiana, Barcelona, 1969, Cité du Vatican-Barcelone, 1972, pp. 5-55, qui montre de profondes connaissances dans le domaine de la sculpture et insiste sur les points communs en Méditerranée. Voir l'analyse des contributions de Noël Duval pour l'Espagne dans G. Ripoll, « Architecture et liturgie en péninsule Ibérique. À propos d'un texte inédit de Noël Duval ", dans Études en hommage à Noël Duval. Villes, palais, églises : recherches récentes sur l'Antiquité tardive, Turnhout, Brepols, Bibliothèque de l'Antiquité tardive, 39 (sous presse).

33. Voir les deux volumes des sarcophages cités précédemment, op. cit. note 2. Manuel Bendala, « In memoriam D. Manuel Sotomayor Muro, S.J. (10.12.1922 - 22.7.2020) ", Madrider Mitteilungen, 61, 2020, pp. 458-462.

34. Catalunya Romànica, sous la direction de Jordi Vigué et Antoni Pladevall, 27 vol., Barcelone, Enciclopèdia Catalana, 1984-1998.

35. P. de Palol et A. Pladevall dir., op. cit. note 29.

36. Xavier Barral i Altet, L'art pre-romànic a Catalunya s. IX-X, Barcelone, Edicions 62, 1981. Sans oublier aussi les corpora à caractère régional tels que Joan Badia i Homs, L'Arquitectura medieval de l'Empordà, I, Baix Empordà, IIA y IIB, Alt Empordà, Gérone, 1977, 1978 et 1981.

37. Enciclopedia del Románico en Asturias, 2 vol., Palencia, Fundación Santa María la Real, 2006.

38. María Cruz Villalón, Mérida visigoda. La escultura arquitectónica y litúrgica, Badajoz, 1985.

39. Isabel Zamorano, "Caracteres del arte visigodo en Toledo ", Anales Toledanos, X, 1974, pp. 7-149.

40. X. Barral i Altet, « La sculpture d'époque visigotique dans la péninsule ibérique », XXXIV Corso di cultura sull'arte ravennate e bizantina, Ravenna 1987, Ravenne, Edizioni del Girasole, 1987, pp. 13-17.

41. X. Barral i Altet, «L'escultura arquitectónica i decorativa en els monuments religiosos de l'antiguitat tardana a Hispània ", II Reunió d'Arqueologia Cristiana Hispànica, Maó 1988, Barcelone, 1994, pp. 41-48.

42. X. Barral i Altet, op. cit. note 41, pp. 41-43.

43. Jean-Marie Hoppe, "Éléments pour une étude de l'esthétique de l'époque visigothique ", Annales d'Histoire de l'Art et d'Archéologie, VII, 1985, pp. 47-72. Id., " Orient-Occident, juifs et chrétiens. À propos de la grande niche du Musée archéologique de Mérida (Badajoz) », Norba-Arte, VII, 1987, pp. 9-46. Id.,1991, op. cit. note 20.

44. J.-M. Hoppe, « Le corpus de la sculpture visigothique. Libre parcours et essai d'interprétation ", dans Visigodos y Omeyas. Un debate entre la Antigüedad tardía y la alta Edad Media, Anejos de Archivo Español de Arqueología, XXIII, 2000, pp. 307-355.

45. Rafaële Mourgues, La sculpture hispanique attribuée à l'époque wisigothique, Université SorbonneParis IV, thèse de Doctorat inédite, Paris, 1996.

46. R. Mourgues, «La sculpture décorative attribuée à l'époque wisigothique en Lusitanie : problématique liée à la production ", dans Économie et territoire en Lusitanie romaine, actes et travaux réunis et présentés par Jean-Gérard Gorges et F. Germán Rodríguez Martín, Madrid, Collection de la Casa de Velázquez, 65, 1999, pp. 525-547.

47. Luis Caballero Zoreda, « Un canal de transmisión de lo clásico en la alta Edad Media española. Arquitectura y escultura de influjo omeya en la Península Ibérica entre mediados del s. VIII e inicios del X », Al-Qantara, 15, 1994, pp. 321-348 et 16, 1995, pp. 107-124.

48. Sally Garen, «Santa María de Melque and Church Construction under Muslim Rule », Journal of the Society of Architectural Historians, 51, 1992, pp. 288-305.

49. M. de los Ángeles Utrero Agudo, Iglesias tardoantiguas y medievales en la Península Ibérica. Análisis arqueológico y sistemas de abovedamiento, Anejos de Archivo Español de Arqueología, XL, 2006. 
50. María Cruz Villalón, «El taller de escultura de Mérida. Contradicciones de la escultura visigoda ", dans Visigodos y Omeyas. Un debate entre la Antigüedad tardía y la alta Edad Media, Anejos de Archivo Español de Arqueología, XXIII, 2000, pp. 265-278. Ead., 2009, op. cit. note 15.

51. Escultura decorativa tardorromana y altomedieval en la Península Ibérica, sous la direction de Luis Caballero et Pedro Mateos, Anejos de Archivo Español de Arqueología, XLI, 2007.

52. Je ne peux pas citer ici toute la bibliographie, mais, je considère comme de bons exemples les références suivantes: M. de los Á. Utrero Agudo, op. cit. note 49. M. de los Ángeles Utrero Agudo, "Las estructuras abovedadas en la historia de la arquitectura hispánica tardoantigua y altomedieval ", Anales de Historia del Arte, vol. extraordinario Cien años de investigación sobre arquitectura medieval española, 2009, pp. 219-232. Et de la même autrice, la défense de cette interprétation proposée dans «Late-Antique and Early Medieval Hispanic Churches and the Archaeology of Architecture: Revisions and Reinterpretation of Constructions, Chronologies and Contexts ", Medieval Archaeology, 54, 2010, pp. 1-33. Isaac Sastre, Los altares de las iglesias hispanas tardoantiguas y altomedievales: estudio arqueológico, Oxford, BAR Int Ser. 2503, 2013. Yiannis Theocharis, "Frühmittelalterliche Architekturdekoration in Spanien: Charakteristika einer "Koine" im Mittelmeerraum », Cahiers Archéologiques, 51, 2003-2004, pp. 29-44.

53. Agustín Azkárate, Gisela Ripoll et Juan Antonio Souto, "Algunas reflexiones personales sobre el simposio "visigodos y omeyas" », dans Visigodos y Omeyas. Un debate entre la Antigüedad tardía y la alta Edad Media, Anejos de Archivo Español de Arqueología, XXIII, 2000, pp. 457-459.

54. Achim Arbeiter, "Alegato por la riqueza del inventario monumental hispanovisigodo », dans Visigodos y Omeyas. Un debate entre la Antigüedad tardía y la alta Edad Media, Anejos de Archivo Español de Arqueología, XXIII, 2000, pp. 249-263. Id., " Die Sakralarchitektur Hispaniens im "Reich von Toledo" aus der Sicht der aktuellen Forschung ", Antiquité tardive, 23, 2015, pp. 219-238.

55. Fedor Schlimbach, «Byzantinische Einflüsse auf den westgotenzeitlichen Kirchenbau in Hispanien? Bemerkungen zur Herleitung der Motive innerhalb der Baudekoration von Santa María de Quintanilla de las Viñas (Burgos) im Streit zwischen Visigotistas und Mozarabistas ", dans Byzanz in Europa. Europas östliches Erbe. Akten des Kolloquiums Byzanz in Europa, sous la direction de Michael Altripp, Turnhout, Brepols, Byzantioc. Studies in Byzantine History and Civilization, 2, 2011, pp. 184-243. Id., San Juan de Baños und der Kirchenbau im westgotischen Königreich von Toledo, Darmstadt, Iberia Archaeologica, 17, 2014.

56. Ripoll et Carrero, op. cit. note 8.

57. Gisela Ripoll, Eduardo Carrero, Daniel Rico, Francesc Tuset, Isabel Velázquez, Aarón López Batlle, Catalina Mas, Montserrat Valls et Miguel Ángel Cau, « La arquitectura religiosa hispánica del siglo IV al X y el proyecto del Corpus Architecturae Religiosae Europeae - CARE-Hispania », Hortus Artium Medievalium, t. 18, 2012, $\mathrm{n}^{\circ}$ 1, pp. 45-73. Voir aussi les résultats remarquables d'un grand projet de recherche pour la Galice dans Jorge Sanjurjo-Sánchez, Rebeca Blanco-Rotea et José Carlos Sánchez-Pardo, "An Interdisciplinary Study of Early Mediaeval Churches in NorthWestern Spain (Galicia) », Heritage, 2, 2019, pp. 599-610, et infra note 96.

58. Daniel Rico Camps, « Arquitectura y epigrafía en la Antigüedad Tardía. Testimonios hispanos ", Pyrenae, t. 40, 2009, n 1, pp. 7-53 ; Id., « Inscripciones monumentales del siglo VIII (de Cangas a Pravia) », Territorio, Sociedad y Poder, 9, 2014, pp. 68-98.

59. Isabel Velázquez, « Epigrafía en la Hispania de época visigoda: nuevas perspectivas, revisiones críticas y estudios ", dans Wisigothica. After M. C. Díaz y Díaz, sous la direction de Carmen Codoñer et Paulo Farmhouse Alberto, Florence, 2014, pp. 307-328. Javier del Hoyo, « A propósito de la inscripción dedicatoria de San Juan de Baños ", dans Temptanda viast. Nuevos estudios sobre la poesía epigráfica latina, sous la direction de C. Fernández Martínez et J. Gómez Pallarés, Bellaterra, UAB, Publicación CD-Rom, 2006 (s/p).

60. Gisela Ripoll et Alexandra Chavarría, « El altar en Hispania, siglos IV al X », Hortus Artium Medievalium, 11, 2005, pp. 29-47. Eduardo Carrero et Daniel Rico, « La organización del espacio litúrgico hispánico entre los siglos VI y XI », Antiquité Tardive, 23, 2015, pp. 239-248. 
61. Paul Reynolds, «Material culture and the economy in the age of Saint Isidore of Seville (6th and 7th centuries) », Antiquité tardive, 23, 2015, pp. 163-210.

62. Alexandra Uscatescu et Juan Carlos Ruiz Souza, " "Orientalismos” y "entanglement" cultural: estímulos y desenfoques historiográficos », Anales de Historia del Arte, 22 (núm. especial II), 2012, pp. 297-308 ; Eid., « El "occidentalismo" de Hispania y la koiné artística mediterránea (siglos VIIVIII)», Goya, 347, 2014, pp. 95-115.

63. Pedro Mateos et Luis Caballero (eds.), Repertorio de arquitectura cristiana en Extremadura: época tardoantigua y altomedieval, Anejos de Archivo Español de Arqueología, XXIX, 2003.

64. L. Caballero et P. Mateos dir., op. cit. note 51.

65. Sergio Vidal, La escultura hispánica figurada de la Antigüedad tardía, Murcie, Corpus Signorum Imperii Romani, España, II/2, 2005.

66. Pour Tolède : Rafael Barroso et Jorge Morín, Regia Sedes Toletana, vol. II, El Toledo visigodo a través de su escultura monumental, Tolède, 2007. Pour Cordoue : Jerónimo Sánchez Velasco, Elementos arquitectónicos de época visigoda en el Museo Arqueológico de Córdoba, Monografías del Museo Arqueológico de Córdoba, 1, Consejería de Cultura de la Junta de Andalucía, Cordoue, 2006 ; Isabel Sánchez Ramos, "Decoración arquitectónica y escultura litúrgica en Corduba », Hortus Artium Medievalium, t. 13, 2007, $\mathrm{n}^{\circ}$ 2, pp. 423-440.

67. Javier Ángel Domingo, Capiteles tardorromanos y visigodos en la península ibérica (siglos IV-VIII d.C.), Tarragone, Institut Català d'Arqueologia Clàssica, Documenta, 13, 2011. Voir aussi une nombreuse bibliographie, dans laquelle il faut souligner : Id., "The use of marble in Hispanic Visigothic Architectural Decoration ", dans ASMOSIA X. Proceedings of the Tenth International Conference Interdisciplinary Studies on Ancient Stone 2012, sous la direction de Patrizio Pensabene et Eleonora Gasparini, Rome, « L’Erma » di Bretschneider, 2015, pp. 527-535, Id., « La reutilización del mármol en la arquitectura tardorromana y visigótica en la península Ibérica», dans Paisajes $e$ historias en torno a la piedra, sous la direction de Virginia García-Entero et al., Madrid, UNED, Monografías de Prehistoria y Arqueología 1, 2020, pp. 319-348.

68. J. Abura, Die westgotenzeitlichen Kapitelle von Toledo, Madrid, Audema Ed., Colección Toledo, Serie Patrimonio, 2014. Pour Segobriga, voir note 5.

69. Nora Büchsenschütz, Iberische Halbinsel und Marokko, Wiesbaden, Dr. Ludwig Reichert Verlag, Repertorium der christlich-antiken Sarkophage 4, 2018.

70. María Cruz Villalón, Beatriz de Griñó et Javier Ángel Domingo, La escultura tardoantigua y altomedieval de Badajoz y Museo Arqueológico Provincial de Badajoz, Cáceres, 2019.

71. Exemplum et Spolia. La reutilización arquitectónica en la transformación del paisaje urbano de las ciudades históricas, sous la direction de Pedro Mateos Cruz et Carlos J. Morán Sánchez, Mérida, MYTRA (Monografías y Trabajos de Arqueología, Instituto de Arqueología), 7, 2020. Il faut aussi tenir compte des publications précédentes dont j'indique seulement celles qui sont pour moi pertinentes : Philippe Araguas, «Spolia/Contrefaçon, deux modalités d'appropriation du passé, pour quelles élites?», Hortus Artium Medievalium, t. 13, 2007, n² 2, pp. 347-358. Spolien im Umkreis der Macht - Spolia en el entorno del poder, sous la direction de Thomas G. Schattner et Fernando Valdés Fernández, Mayence, Iberia Archaeologica, 12, 2009, ainsi que Josep Maria Gurt i Esparraguera et Pilar Diarte Blasco, « Spolia et Hispania: alcuni esempi peninsulari», Hortus Artium Medievalia, t. 17, 2011, pp. 7-22. José Carlos Sánchez-Pardo, « El reuso de materiales y estructuras antiguas en las iglesias altomedievales de Galicia. Casos, problemas y motivaciones ", Estudos do Quaternário, 12, Braga, APEQ, 2015, pp. 95-110.

72. Toujours utile : A New Companion to Digital Humanities, sous la direction de Susan Schreibman, Ray Siemens et John Unsworth, Chichester, Wiley-Blackwell, Blackwell Companions to Literature and Culture, 2016 et l'état de la question posée, pour la période médiévale, des sources textuelles à la matérialité archéologique et architecturale : David J. Birnbaum, Sheila Bonde et Mike Kestemont, «The Digital Middle Ages: An Introduction », Speculum, t. 92, 2017, n 1, pp. 1-38. Il est intéressant de lire l'évaluation sur les humanités numériques (en particulier l'histoire de l'art) 
de Johanna Drucker, «Is There a "Digital” Art History? », Visual Resources, 29.1/2, 2013, pp. 5-13 ; bien que datant de 2013, c'est une bonne réflexion.

73. G. Ripoll, « Corpus Architecturae Religiosae Europeae, saec. IV-X (CARE-Hispania) », Mainake, 31, 2009, pp. 229-242. G. Ripoll et al., op. cit. note 57. Voir aussi http://www.carehispania.info/ portal/

74. Gian Pietro Brogiolo et Miljenko Jurković, « Corpus Architecturae Religiosae Europeae (IV-X saec.). Introduction », Hortus Artium Medievalium, t. 18, 2012, nº 1, pp. 7-26.

75. Cf. notes 57 et 73 (supra).

76. Pour les Baléares une thèse a été dirigée par moi-même et M. A. Cau, cf. Silvia Alcaide, Arquitectura cristiana balear en la antigüedad tardía (ss. V-X d.C.), Universitat Rovira i Virgili, Tarragone, 2011. http://hdl.handle.net/10803/32933 [Date de consultation : 24.07.2021].

77. Pascale Chevalier, Ludovic Granjon, Éric Leclercq, Arnaud Millereux, Marinette Savonnet et Christian Sapin, « Base de Données annotées et Wiki pour la constitution du corpus numérique CARE ", Hortus Artium Medievalium, t. 18, 2012, n 1, pp. 27-35. La même équipe a fait une proposition qui n'a pas été utilisée : Ludovic Granjon, Pascale Chevalier, Éric Leclercq, Arnaud Millereux, Laure Saligny, Marinette Savonnet et Christian Sapin, « Application cartographique en ligne du projet CARE : principes et fonctionnement », Hortus Artium Medievalium, t. 18, 2012, $\mathrm{n}^{\circ} 1$, pp. 37-43.

78. AEHTAM : http://hesperia.ucm.es/consulta_aehtam/web_aehtam/ [Date de consultation : 22.07.2021].

79. http://www.ascorpus.ac.uk/index.php [Date de consultation : 23.07.2021].

80. Rocío Gutiérrez, Mercedes Farjas et Isabel Velázquez, «EPIARQ: A Spatial Data Infrastructure for Research in Medieval Epigraphy ", Hortus Artium Medievalium, t. 21, 2015, pp. 324-334.

81. Nous avons tenté plusieurs appels à projets, car le coût économique est très élevé, mais nous devrons attendre de nouvelles opportunités.

82. 3D recording, documentation and management of cultural heritage, sous la direction d'Efstratios Stylianidis et Fabio Remondino, Dunbeath, Whittles Publishing, 2016 ; ainsi que Jeroen De Reu, Philippe De Smedt, Davy Herremans, Marc Van Meirvenne, Pieter Laloo et Wim De Clercq, « On introducing an image-based 3D reconstruction method in archaeological excavation practice ", Journal of Archaeological Science, 41, 2014, pp. 251-262.

83. Suggestif et précis sur les possibilités offertes par les méthodes analytiques pour comprendre la séquence chrono-spatiale d'un bâtiment : Jorge Sanjurjo-Sánchez, « Dating historical buildings: an update on the possibilities on absolute dating methods ", International Journal of Architectural Heritage, t. 10, 2016, $\mathrm{n}^{\circ}$ 5, pp. 620-635.

84. Justin Leidwanger, Elizabeth S. Greene et Andrew Donnelly, " The Sixth-Century CE Shipwreck at Marzamemi ", American Journal of Archaeology, t. 125, 2021, n² 2, pp. 283-317, citant la bibliographie précédente, notamment celle de Gerhard Kapitän, auteur de la découverte dès les années 1960.

85. L'étude détaillée est encore en attente, mais on peut déjà consulter : María Belén, Manuel Fernández-Miranda, Damià Cerdà et Joan C. de Nicolás, Arqueologia submarina en Menorca, Madrid, Fundación Juan March, 1977 (voir pp. 95-102) ; Manuel Fernández-Miranda, Alicia Rodero Riaza, « El yacimiento submarino de Favaritx (Menorca) ", VI Congreso Internacional de Arqueología Submarina, Cartagena 1982, Madrid, 1985, pp. 175-188.

86. Mathias Bruno, Donato Attanasio, Walter Prochaska et Ali Bahadir Yavuz, « An update on the use and distribution of white and black Göktepe marbles from the first century AD to late antiquity ", dans ASMOSIA X. Proceedings of the Tenth International Conference Interdisciplinary Studies on Ancient Stone 2012, sous la direction de Patrizio Pensabene et Eleonora Gasparini, Rome, " L'Erma » di Bretschneider, 2015, pp. 461-468.

87. Javier Arce, " Mercados rurales (nundinae) en la Hispania tardorromana ", dans Homenatge a M. Tarradell, Barcelone, Curial Edicions Catalanes, 1993, pp. 867-871. Précédé par la publication de 
la fouille du site : Javier Arce, Luis Caballero et Miguel Ángel Elvira, «El edificio octogonal de Valdetorres de Jarama (Madrid) », dans Congreso Internacional "La Hispania de Teodosio", Ségovie, 1997, vol. 2, pp. 321-337. Voir les considérations à propos des sculptures des villae dans G. Ripoll, « Aristocratic residences in Late Antique Hispania ", dans Roman Villas in the Mediterranean Basin: Late Republic to Late Antiquity, sous la direction d'Annalisa Marzano et Guy P. R. Métraux, New York, Cambridge University Press, 2018, pp. 426-452 (voir pp. 441-443).

88. Marmora Hispana: explotación y uso de los materiales pétreos en la Hispania romana, sous la direction de Trinidad Nogales Basarrate et José Beltrán Fortes, Rome, " L'Erma » di Bretschneider, 2008. El marmor en Hispania. Explotación, uso y difusión en época romana, sous la direction de Virginia García-Entero, Madrid, Universidad Nacional de Educación a Distancia, 2012.

89. M. Pilar Lapuente, Bruno Turi et Philippe Blanc, " Marbles from Roman Hispania: stable isotope and cathodoluminescence characterization ", Applied Geochemistry, 15, 2000, pp. 1469-1493. J. M. P. Cabral, M. C. R. Vieira, P. M. Carreira, M. O. Figueiredo, T. P. Penam et A. Tavares, «Preliminary study on the isotopic and chemical characterization of marbles from Alto Alentejo (Portugal) », Ancient Stones: Quarrying, Trade and Provenance, sous la direction de Marc Waelkens, Norman Herz et Luc Moens, Louvain, Leuven University Press, 1992, pp. 191-198. Voir aussi Irene Mañas Romero, « Marmora de las canteras de Estremoz, Alconera y Sintra: su uso y difusión ", dans El marmor en Hispania: explotación, uso y difusión en época romana, sous la direction de Virginia García-Entero, Madrid, UNED, 2012, pp. 331-346.

90. Sergio Vidal Álvarez et Virginia García-Entero, « The use of Estremoz marble in late antique sculpture of Hispania: new data from the petrographic and cathodoluminescence analyses ", dans ASMOSIA X. Proceedings of the Tenth International Conference Interdisciplinary Studies on Ancient Stone 2012, sous la direction de Patrizio Pensabene et Eleonora Gasparini, Rome, " L’Erma » di Bretschneider, 2015, pp. 355-366. Sergio Vidal Álvarez, Virginia García-Entero et Anna Gutiérrez García-Moreno, "La utilización del mármol de Estremoz en la escultura hispánica de la antigüedad tardía: los sarcófagos », DigitAR. Digital Journal of Archaeology, Architecture and Arts, 3, 2016, pp. 119-128. Pour les questions épigraphiques du sarcophage, voir : Archivo Epigráfico de Hispania Tardoantigua y Medieval / AEHTAM, $\mathrm{n}^{\circ}$ 1748, consultable en ligne : http://hesperia.ucm.es/ consulta_aehtam/Generalidades.php?filtrar=\&valor=\&id=1783 [Date de consultation : 22.07.2021]. Et l'étude du sarcophage dans son contexte, dans Begoña Torre Miguel, « The sarcophagus of Ithacio and the royal pantheon of Oviedo ", Hortus Artium Medievalium, t. 24, 2018, pp. 329-334.

91. Sergio Vidal Álvarez, "Análisis arqueométricos del sarcófago de Pueblanueva (Toledo) y estudio de cinco fragmentos de sarcófago procedentes de Pueblanueva en las colecciones del Museo Arqueológico Nacional ", Boletín del Museo Arqueológico Nacional, 34, 2016, pp. 195-210.

92. Carmen Fernández Ochoa, Manuel Bendala Galán, Virginia García-Entero et Sergio Vidal Álvarez, "Cubierta de sarcófago con el ciclo de Jonás hallada en Carranque (Toledo) », Archivo Español de Arqueología, 84, 2011, pp. 231-242. Virginia García-Entero et Sergio Vidal Álvarez, « El uso del mármol en el yacimiento de Carranque (Toledo) ", dans El marmor en Hispania. Explotación, uso y difusión en época romana, sous la direction de Virginia García-Entero, Madrid, UNED, pp. 135-154.

93. Silvia González Soutelo, Anna Gutiérrez García-Moreno et Hernando Royo Plumed, «El sarcófago romano de Tui (Pontevedra): un ejemplo de la presencia de material marmóreo foráneo en el noroeste de la península Ibérica », SPAL, t. 27, 2018, n 2, pp. 229-246.

94. Pour la discussion historiographique, voir H. Schlunk, « Un taller de sarcófagos cristianos en Tarragona ", Archivo Español de Arqueología, 24, 1951, pp. 67-97. Pere de Palol, « El taller de sarcófagos de Tarragona ", VIII Corso di Cultura sull'Arte Ravennate e Bizantina, 1961, pp. 219-226. H. Schlunk, "Sarkophage aus christlichen Nekropolen in Karthago und Tarragona ", Madrider Mitteilungen, 8, 1967, pp. 230-258 ; Id., "Sarcófagos paleocristianos labrados en Hispania ", Actas del VIII Congreso Internacional de Arqueología Cristiana (Barcelona 1969), Cité du Vatican-Barcelone, 
1972, pp. 187-218. En ce qui concerne les analyses pétrographiques, voir en dernier lieu avec la bibliographie antérieure : Isabel Rodà, « Los sarcófagos importados de Cartago en Tarraco. Un inventario de los manufacturados en "kadel" ", dans Iconographie funéraire romaine et société : corpus antique, approches nouvelles ?, sous la direction de Martin Galinier et François Baratte, Perpignan, Presses Universitaires de Perpignan, Collection Histoire de l'art, 2013, pp. 193-202.

95. Une vue d'ensemble à propos des importations des ateliers romains et des ateliers locaux dans G. Ripoll, « Sarcófagos de la Antigüedad tardía hispánica: importaciones y talleres locales », Antiquité tardive, 1, 1993, pp. 153-158.

96. Aureli Àlvarez, Virginia García-Entero, Anna Gutiérrez et Isabel Rodà, El marmor de Tarraco: explotació, utilització i comercialització de la pedra de Santa Tecla en època romana, Tarragone, Institut Català d'Arqueologia Clàssica, Hic et Nunc, 6, 2009 (voir pp. 89-93).

97. Antonel Jepure, Das westgotenzeitliche Gräberfeld von Madrona (Segovia, Spanien), JuliusMaximilians-Universität zu Würzburg et Universidad Autónoma de Madrid, thèse de Doctorat inédite, Würzburg-Madrid, 2006 (voir pp. 48-63).

98. Voir l'ensemble des résultats dans J. Sanjurjo-Sánchez, R. Blanco-Rotea et J. C. Sánchez-Pardo, op. cit. note 57. Voir aussi l'étude centrée sur l'église de Santa Comba de Bande : José Carlos Sánchez-Pardo, Rebeca Blanco-Rotea et Jorge Sanjurjo-Sánchez, «The church of Santa Comba de Bande and early medieval Iberian architecture - new chronological results ", Antiquity, t. 91, 2017, $\mathrm{n}^{\circ}$ 358, pp. 1011-1026. L'approche de la réutilisation des matériaux est d'un grand intérêt, voir J. C. Sánchez-Pardo, 2015, op. cit. note 71. Et l'article déjà mentionné sur les possibilités des méthodes d'analyse de J. Sanjurjo-Sánchez, op. cit. note 83.

99. Pour les résultats des fouilles précédentes : Manuel Lechuga, Rafael González et Francisco Fernández, "Un recinto de planta absidal en el yacimiento de Los Villaricos, Mula, Murcia », Antigüedad y Cristianismo, XXI, 2004, pp. 171-181 ; Rafael González, Francisco Fernández et José Antonio Zapata, « La villa romana de Los Villaricos (Mula, Murcia): un gran centro productor de aceite en la Hispania Tarraconense ", Archivo Español de Arqueología, 91, 2018, pp. 89-113. Voir la nouvelle du 16 juillet 2021 avec des photos dans Localizado un sarcófago visigodo en la villa romana de Los Villaricos de Mula, https://www.laopiniondemurcia.es/municipios/2021/07/16/localizadosarcofago-visigodo-villa-romana-55113661.html et celle du 19 juillet 2021 dans The New York Times, Perfectly preserved Visigoth coffin unearthed from Roman ruins in southern Spainhttps:// www.thetimes.co.uk/article/perfectly-preserved-visigoth-coffin-unearthed-from-roman-ruinsin-southern-spain-h52qrsxp3

\section{RÉSUMÉS}

La sculpture de l'Antiquité tardive et du haut Moyen Âge en péninsule Ibérique ne possède pas un corpus complet, mais un grand nombre d'études dispersées qui permettent d'établir un état de la question. Nous disposons de corpora pour des régions, villes ou collections de musées, comme Badajoz, Cordoue, Tarragone ou Tolède, ainsi que des répertoires à caractère thématique, comme la sculpture figurée, les autels liturgiques ou la sculpture funéraire. Cela dit, il manque des études spécifiques sur les spolia de prestige concernant la sculpture et l'épigraphie en les distinguant du matériel à caractère exclusivement constructif, ainsi que de bonnes analyses sur le mobilier liturgique, les ateliers locaux, les importations et une bonne séquence chronologique des productions. L'objectif de cette présentation est d'offrir un état de la connaissance sur ce 
matériel, sur l'iconographie et la production, en vue de cerner des mécanismes de travail futur, tout spécialement des analyses archéométriques.

Late Antique and Early Medieval sculpture in the Iberian Peninsula does not have a complete corpus, but a large number of dispersed studies that allow us to establish a state of the art. We have corpora for regions, cities or museum collections, such as Badajoz, Córdoba, Tarragona or Toledo, as well as thematic directories, such as figurative sculpture, liturgical altars or funerary sculpture. However, specific studies on the prestige spolia concerning sculpture and epigraphy are lacking, distinguishing them from material of an exclusively constructive nature, as well as good analyses of liturgical furniture, local workshops, imports and a good chronological sequence of productions. The objective of this presentation is to offer a state of knowledge on this material, on iconography and production, in order to identify future work mechanisms, especially archaeometric analysis.

\section{INDEX}

Mots-clés : architecture, décor sculpté, sculptures, mobilier liturgique, sarcophages, ateliers, commanditaires, Antiquité tardive, haut Moyen Âge, IVe - Xe siècle

Keywords : architecture, sculpted decoration, sculptures, liturgical furniture, sarcophagi, workshops, clients and consumers, Late Antiquity, Early Middle Ages, 4th - 10th centuries

\section{AUTEUR}

\section{GISELA RIPOLL}

Gisela Ripoll est Professeur d'Archéologie à l'Université de Barcelone et fait partie du laboratoire ERAAUB/Equip de Recerca Arqueològica i Arqueomètrica, Universitat de Barcelona

(2017SGR1043). Elle a été formée par des pionniers, à Barcelone auprès de P. de Palol et à Paris 4 de Noël Duval, en s'intéressant et focalisant sa recherche en archéologie, architecture et histoire de l'Antiquité tardive et du haut Moyen Âge en péninsule Ibérique, depuis les transformations qui ont eu lieu dans les régions urbaines et rurales à la suite de la pénétration du christianisme jusqu'à la caractérisation de l'arrivée et de l'installation des peuples barbares. Elle a été professeur invité à l'Université de Paris-Sorbonne, Paris 4, l'École Normale Supérieure et l'École Pratique des Hautes Études (IV ${ }^{\text {e }}$ Section), ainsi que Senior Researcher à l'Institute for Advanced Study de Princeton (USA). Parmi les projets récents, il convient de citer, les fortifications de Carcassonne, le Corpus architecturae religiosae Europeae (s. IV-X)-CARE-Hispania, ainsi que Ecclesiae, coemeteria et loci (s. VIII-XI)/Églises, cimetières et habitats avec la fouille de trois sites majeurs : Olèrdola, Sidillà, Sant Quirze de Colera et, enfin, Sant Hilari d'Abrera, dans le territoire situé au sud de Barcelone, un site romain, de l'Antiquité tardive et médiéval. Ces contributions à la recherche lui ont fait mériter le Prix Raoul Duseigneur octroyé par l'Institut de France (Académie des Inscriptions et Belles Lettres) aux hispanistes.

Gisela Ripoll is professor of archaeology at the Universitat de Barcelona and part of the ERAAUB/ Equip de Recerca Arqueològica i Arqueomètrica (2017SGR1043) there. She was educated by pioneers in their fields: P. de Palol in Barcelona and Noël Duval at Université de Paris 4. Her research has focused on the archaeology, architecture and history of the Iberian peninsula in Late Antiquity and the Early Middle Ages, from the transformations that took place in urban and rural regions after the penetration of Christianity to the characterisation of the arrival and installation of barbarian peoples. She has been a guest professor at the Université de Paris- 
Sorbonne, the École Normale Supérieure and the École Pratique des Hautes Études (Sciences Historiques et Philologiques), and senior researcher the Institute for Advanced Study, Princeton. Recent projects include the fortifications of Carcassonne, the Corpus architecturae religiosae Europeae (s. IV-X)-CARE-Hispania, and Ecclesiae, coemeteria et loci (s. VIII-XI) with the excavation of three major sites: Olèrdola, Sidillà, Sant Quirze de Colera and Sant Hilari d'Abrera south of Barcelona, a Roman and early medieval site. These contributions to research have earned her the Prix Raoul Duseigneur, which the Institut de France (Académie des Inscriptions et Belles Lettres) to Hispanists. 